\title{
RESEARCH
}

\section{Modified transmission eigenvalues for inverse scattering in a fluid-solid interaction problem}

\author{
Peter Monk ${ }^{1}\left[\right.$ and Virginia Selgas ${ }^{2 *}$ (1)
}

\section{*Correspondence:}

selgasvirginia@uniovi.es

2 Departamento de Matemáticas, Universidad de Oviedo, EPIG, C/ Luis Ortiz Berrocal s/n, 33203 Gijón, Spain

Full list of author information is available at the end of the article

\begin{abstract}
Target signatures are discrete quantities computed from measured scattering data that could potentially be used to classify scatterers or give information about possible defects in the scatterer compared to an ideal object. Here, we study a class of modified interior transmission eigenvalues that are intended to provide target signatures for an inverse fluid-solid interaction problem. The modification is based on an auxiliary problem parametrized by an artificial diffusivity constant. This constant may be chosen strictly positive, or strictly negative. For both choices, we characterize the modified interior transmission eigenvalues by means of a suitable operator so that we can determine their location in the complex plane. Moreover, for the negative sign choice, we also show the existence and discreteness of these eigenvalues. Finally, no matter the choice of the sign, we analyze the approximation of the eigenvalues from far field measurements of the scattered fluid pressure and provide numerical results which show that, even with noisy data, some of the eigenvalues can be determined from far field data.
\end{abstract}

Keywords: Fluid-solid interaction, Inverse scattering, Steklov eigenvalues, Modified interior transmission eigenvalues

Mathematics Subject Classification: 35R30, 35P25, 35P05, 65N21, 65N25

\section{Introduction}

Target signatures are discrete data that can be computed from the wave field scattered by an unknown target. It has been proposed that these discrete data can be used either to categorize the scatterer by comparing the signature to a dictionary of known signatures, or to detect changes in the structure of a scatterer. The first target signatures to be proposed were for electromagnetic waves and could be computed from time domain measurements of a radar signal [23]. In recent years, the quest for target signatures for inverse acoustic and electromagnetic scattering has been centered around three eigenvalue problems: transmission eigenvalues, Steklov type eigenvalues and modified interior transmission eigenvalues. The first of these, transmission eigenvalues, arise in the study of the injectivity of the far field operator for a bounded penetrable scatterer. However, transmission eigenvalues can only be detected by scanning in frequency and are restricted

(c) The Author(s) 2021. This article is licensed under a Creative Commons Attribution 4.0 International License, which permits use, sharing, adaptation, distribution and reproduction in any medium or format, as long as you give appropriate credit to the original author(s) and the source, provide a link to the Creative Commons licence, and indicate if changes were made. The images or other third party material in this article are included in the article's Creative Commons licence, unless indicated otherwise in a credit line to the material. If material is not included in the article's Creative Commons licence and your intended use is not permitted by statutory regulation or exceeds the permitted use, you will need to obtain permission directly from the copyright holder. To view a copy of this licence, visit http://creativecommons.org/licenses/by/4.0/. 
to non-absorbing media. These are practical limitations. For a discussion of these eigenvalues, see [10].

In order to obtain signatures that can be determined for absorbing media, and which only require scattering data at a single frequency, Cakoni et al. [4] proposed to use the so-called Steklov eigenvalues for the Helmholtz equation as target signatures. This study was extended to Maxwell's equations in [5], and to the fluid-solid interaction problem in [20]. For the fluid-solid problem, Steklov type eigenvalues (termed impedance eigenvalues in [20]) can be determined from scattering data, but only a few can be determined from such data.

In order to provide a tunable parameter that can help increase the sensitivity of the eigenvalues to changes in the scatterer, Cogar et al. [8] introduced modified transmission eigenvalues. This idea was recently extended to Maxwell's equations in [9].

In our paper, we develop the modified interior transmission eigenvalues for the fluidsolid interaction problem. Such eigenvalues involve a modified interior transmission problem (mITP) with a coupling between the fluid and elastic wave fields, and results in a discrete set of eigenvalues. The properties of these eigenvalues depend on the choice of an artificial diffusivity parameter that can be positive (the natural case [8]) or negative (the metamaterial case [2]). The idea of using a metamaterial was first suggested in [2] in the context of the Helmholtz equation. In particular for the metamaterial case, it is possible to prove monotonicity results for the eigenvalues which may be helpful in characterizing the elastic properties of the scatterer. We point out that only physical data from a standard linear elastic scatterer are used to determine these eigenvalues, regardless of the sign of the artificial diffusivity.

Among the main contributions of this paper is to give the first study of modified interior transmission eigenvalues for the fluid-solid problem. This is also the first such study of modified interior transmission eigenvalues for a multi-physics problem and shows that this type of eigenvalues can be used in this case.

To understand the behavior of the eigenvalues associated with the mITP, we reformulate the mITP first by means of an auxiliary boundary operator. We then study the mITP by superposition with a reference case, and this reasoning allows us to make use of the Fredholm alternative. The behavior of modified interior transmission eigenvalues depends on the sign of an auxiliary parameter defining the modified far field operator. In particular, when this parameter is negative, we prove an upper bound; and, this allows us to guarantee the existence of a reference case and use it to show the existence of infinitely many discrete eigenvalues. Finally, for either sign choice, we study the approximation of these eigenvalues using a modified far field equation.

The outline of this paper is as follows. First, in Sect. 2, we give a brief summary of the forward problem, drawing heavily on the presentation in [20]. In Sect. 3, we define the modified interior transmission eigenvalues, motivating their choice by considering the injectivity of the modified far field operator defined at the start of that section. We also derive theorems concerning the discreteness and distribution of modified interior transmission eigenvalues. Next, in Sect. 4 we demonstrate the usual theorems concerning the approximation of modified transmission eigenvalues using solutions of the far field equation. Then, in Sect. 5 we show a few numerical examples to illustrate our theory. Finally, we end with conclusions and comments on further study. 
We perform the analysis for a bounded elastic scatterer in $\mathbb{R}^{m}$ for $m=2,3$. Generally, bold face quantities denote vectors as well as spaces of vector-valued fields: For example, $\boldsymbol{u}(\boldsymbol{x})$ denotes the displacement of the solid at the point $\boldsymbol{x} \in \mathbb{R}^{m}$; besides, if $\mathcal{O}$ is a bounded open set, then $H^{1}(\mathcal{O})$ stands for the standard Sobolev space of functions with $L^{2}$-derivatives, and $\boldsymbol{H}^{1}(\mathcal{O})=\left(H^{1}(\mathcal{O})\right)^{m}$.

\section{The forward fluid-solid interaction problem}

This section follows closely the corresponding introductory material in [20]. For this reason, we only give enough detail to define the basic notation, and direct the reader to that paper for more details.

We first recall a standard mathematical formulation of the forward fluid-solid interaction problem, cf. [16]. We consider a linear elastic body that occupies a bounded domain $\Omega \subset \mathbb{R}^{m}$. A fluid occupies the unbounded domain $\Omega_{e}=\mathbb{R}^{m} \backslash \bar{\Omega}$, which is assumed to be connected. We also assume that the boundary $\Gamma=\partial \Omega$ is smooth and denote by $\boldsymbol{n}$ the unit normal on $\Gamma$ outward to $\Omega$.

As usual, we denote by $\epsilon(\boldsymbol{u})=\left(\frac{1}{2}\left(\partial_{i} u_{j}+\partial_{j} u_{i}\right)\right)_{i, j=1}^{m}$ the strain tensor and by $\sigma(\boldsymbol{u})=$ $\lambda \operatorname{div} \boldsymbol{u} I+2 \mu \epsilon(\boldsymbol{u})$ the stress tensor, where $I$ is the $m \times m$ identity tensor. The elastic coefficients $\lambda, \mu \in \mathbb{R}$ are the Lamé moduli and are assumed to satisfy (cf. [21]):

$$
\mu>0, \quad \lambda+\frac{2}{m} \mu>0 .
$$

The wavenumber in the fluid is denoted by $k=\omega / c_{f} \in \mathbb{R}, k>0$, where $\omega$ is the angular frequency of the fields and $c_{f}$ is the speed of sound in the fluid. In addition, the mass densities in the fluid $\left(\rho_{f}\right)$ and the solid $\left(\rho_{s}\right)$ are positive, and the mass density in the fluid $\left(\rho_{f}\right)$ is constant.

We shall consider incident plane waves from the fluid on the solid:

$$
p^{i}(\boldsymbol{x})=p^{i}(\boldsymbol{x}, \boldsymbol{d})=\mathrm{e}^{\imath k \boldsymbol{x} \cdot \boldsymbol{d}} \quad \text { in } \mathbb{R}^{m}
$$

where $\boldsymbol{d} \in \mathbb{S}^{m-1}=\left\{\boldsymbol{x} \in \mathbb{R}^{m} ;|\boldsymbol{x}|=1\right\}$ is the direction of propagation.

The standard traction operator is

$$
\boldsymbol{T} \boldsymbol{u}=\sigma(\boldsymbol{u}) \boldsymbol{n}=\lambda \operatorname{div} \boldsymbol{u} \boldsymbol{n}+2 \mu \epsilon(\boldsymbol{u}) \boldsymbol{n} \quad \text { on } \Gamma .
$$

Then, the elastodynamic displacement field $\boldsymbol{u} \in \boldsymbol{H}^{1}(\Omega)$ and the dynamic component of the fluid pressure $p \in H_{\text {loc }}^{1}\left(\Omega_{e}\right)$ solve the following fluid-solid interaction problem:

$$
\left.\begin{array}{ll}
\nabla \cdot \sigma(\boldsymbol{u})+\rho_{s} \omega^{2} \boldsymbol{u}=\mathbf{0} & \text { in } \Omega, \\
\begin{array}{ll}
\Delta p+k^{2} p=0 & \text { in } \Omega_{e}, \\
\boldsymbol{T} \boldsymbol{u}=-p \boldsymbol{n} & \text { on } \Gamma, \\
\boldsymbol{u} \cdot \boldsymbol{n}=\frac{1}{\rho_{f} \omega^{2}} \frac{\partial p}{\partial \boldsymbol{n}} & \text { on } \Gamma, \\
p=p^{i}+p^{s} & \text { and }
\end{array} \quad \frac{\partial p^{s}}{\partial r}-\imath k p^{s}=\mathrm{O}\left(r^{-(m-1) / 2}\right) \text { as } r=|\boldsymbol{x}| \rightarrow \infty .
\end{array}\right\}
$$

For a complete description of the derivation of this model see [16] or [21], and the references therein. 
To define conditions under which problem (2.3) has a unique solution, we recall that $\omega$ is a Jones frequency with Jones mode $\boldsymbol{u} \in \boldsymbol{H}^{1}(\Omega)$ whenever $\boldsymbol{u}$ is a non-trivial solution of the following interior problem:

$$
\left.\begin{array}{rlrl}
\nabla \cdot \sigma(\boldsymbol{u})+\rho_{s} \omega^{2} \boldsymbol{u} & =\mathbf{0} & & \text { in } \Omega, \\
\boldsymbol{T} \boldsymbol{u} & =\mathbf{0} & & \text { on } \Gamma, \\
\boldsymbol{u} \cdot \boldsymbol{n} & =0 & & \text { on } \Gamma .
\end{array}\right\}
$$

Such modes (if any) are discrete. They exist, for example, when $\Omega$ is a disk in $\mathbb{R}^{2}$ or a ball in $\mathbb{R}^{3}$. By the Fredholm alternative, problem (2.3) is well-posed when $\omega$ is not a Jones frequency (see, e.g., $[16,21,24])$ and from now on we shall assume that $\omega$ is not a Jones mode.

Because $p^{s}$ is a radiating solution of the Helmholtz equation, it has the asymptotic expansion

$$
p^{s}(\boldsymbol{x})=\frac{\mathrm{e}^{l k r}}{r^{(m-1) / 2}} p^{\infty}(\widehat{\boldsymbol{x}})+\mathrm{O}\left(r^{-(m+1) / 2}\right) \quad \text { as } r=|\boldsymbol{x}| \rightarrow \infty,
$$

see [10]. Here, the function $p^{\infty}$, called the far field pattern of the scattered field, is given by

$$
p^{\infty}(\widehat{\boldsymbol{x}})=-c_{m} \int_{\Gamma}\left(\imath k p^{s}(\boldsymbol{y}) \boldsymbol{n}(\boldsymbol{y}) \cdot \widehat{\boldsymbol{x}}+\frac{\partial p^{s}}{\partial \boldsymbol{n}}(\boldsymbol{y})\right) \mathrm{e}^{-\imath k \boldsymbol{y} \cdot \widehat{\boldsymbol{x}}} \mathrm{dS}_{\boldsymbol{y}},
$$

where

$$
c_{m}= \begin{cases}\frac{\mathrm{e}^{\imath \pi / 4}}{\sqrt{8 \pi k}} & \text { if } m=2, \\ \frac{1}{4 \pi} & \text { if } m=3 .\end{cases}
$$

Given an incident plane wave as in (2.2), we denote by $\boldsymbol{u}(\cdot, \boldsymbol{d}), p^{s}(\cdot, \boldsymbol{d})$ and $p^{\infty}(\cdot, \boldsymbol{d})$ the associated displacement field, scattered wave and far field pattern, respectively.

A key tool in inverse scattering is the far field operator $F: L^{2}\left(\mathbb{S}^{m-1}\right) \rightarrow L^{2}\left(\mathbb{S}^{m-1}\right)$ defined by (see [10])

$$
(F g)(\widehat{\boldsymbol{x}})=\int_{\mathbb{S}^{m-1}} p^{\infty}(\widehat{\boldsymbol{x}}, \boldsymbol{d}) g(\boldsymbol{d}) \mathrm{d} \mathrm{S}_{\boldsymbol{d}} \quad \text { for all } \widehat{\boldsymbol{x}} \in \mathbb{S}^{m-1} .
$$

By the linearity of the forward problem (2.3), $F g=p_{g}^{\infty}$ where $p_{g}^{\infty}$ is the far field pattern of the scattered wave due to the incident field given by the Herglotz wave function

$$
p_{g}^{i}(\boldsymbol{x})=\int_{\mathbb{S}^{m-1}} \mathrm{e}^{\imath k \boldsymbol{x} \cdot \boldsymbol{y}} g(\boldsymbol{y}) \mathrm{dS}_{\boldsymbol{y}}=\int_{\mathbb{S}^{m-1}} p^{i}(\boldsymbol{x}, \boldsymbol{y}) g(\boldsymbol{y}) \mathrm{dS}_{\boldsymbol{y}} .
$$

\section{Modified interior transmission eigenvalues}

In this section, we define modified transmission eigenvalues for the fluid-solid problem. In order to relate them to a scattering problem, we propose a modification of the far field operator $F$ motivated by the corresponding theory for the Helmholtz equation in [8]. 
More precisely, given parameters $\gamma \neq 0$ and $\beta \in \mathbb{C}$, we consider the auxiliary transmission problem of finding $h \in H_{\text {loc }}^{1}\left(\mathbb{R}^{m}\right)$ such that

$$
\begin{aligned}
& \frac{1}{\gamma} \Delta h+\beta k^{2} h=0 \quad \text { in } \Omega \text {, } \\
& \Delta h+k^{2} h=0 \quad \text { in } \Omega_{e} \text {, } \\
& h^{+}-h^{-}=0 \quad \text { on } \Gamma \text {, } \\
& \frac{\partial h^{+}}{\partial \boldsymbol{n}}-\frac{1}{\gamma} \frac{\partial h^{-}}{\partial \boldsymbol{n}}=0 \quad \text { on } \Gamma \text {, } \\
& h=h^{i}+h^{s} \text { and } \frac{\partial h^{s}}{\partial r}-\imath k h^{s}=\mathrm{O}\left(r^{-(m-1) / 2}\right) \text { as } r \rightarrow \infty .
\end{aligned}
$$

Here, $h^{ \pm}$denotes the trace of the field from the exterior or the interior of $\Omega$, respectively; similarly, $\frac{\partial h^{ \pm}}{\partial \boldsymbol{n}}$ stands for the normal derivatives taken from the exterior or the interior, respectively. From now on, we assume that $\gamma \neq 0$ is fixed and $\beta \in \mathbb{C}$ is the parameter we may change, and which will ultimately become an eigenvalue. This problem is well-posed when $\gamma>0$ for any $\beta \in \mathbb{C}_{+}=\{z \in \mathbb{C} ; \operatorname{Im}(z) \geq 0\}$, see [10]; it is also well-posed in case $\gamma<0$ when $\gamma \neq-1$ and $\beta \in \mathbb{C}_{+}$, see $[13,29]$.

We consider an incident plane wave $h^{i}=p^{i}(\cdot, \boldsymbol{d})$ as in $(2.2)$, and denote by $h^{s}(\cdot, \boldsymbol{d})$ and $h^{\infty}(\cdot, \boldsymbol{d})$ the associated scattered wave and its far field pattern, respectively. We introduce $F_{\beta}: L^{2}\left(\mathbb{S}^{m-1}\right) \rightarrow L^{2}\left(\mathbb{S}^{m-1}\right)$ the far field operator associated with this auxiliary problem, which is given by

$$
\left(F_{\beta} g\right)(\widehat{\boldsymbol{x}})=\int_{\mathbb{S}^{m-1}} h^{\infty}(\widehat{\boldsymbol{x}}, \boldsymbol{d}) g(\boldsymbol{d}) \mathrm{d} \mathrm{S}_{\boldsymbol{d}} \quad \text { a.e. } \boldsymbol{d} \in \mathbb{S}^{m-1} .
$$

We then define the associated modified far field operator $\mathcal{F}_{\beta}=F-F_{\beta}: L^{2}\left(\mathbb{S}^{m-1}\right) \rightarrow$ $L^{2}\left(\mathbb{S}^{m-1}\right)$, that is

$$
\left(\mathcal{F}_{\beta} g\right)(\widehat{\boldsymbol{x}})=\int_{\mathbb{S}^{m-1}}\left(p^{\infty}(\widehat{\boldsymbol{x}}, \boldsymbol{d})-h^{\infty}(\widehat{\boldsymbol{x}}, \boldsymbol{d})\right) g(\boldsymbol{d}) \mathrm{d} \mathrm{S}_{\boldsymbol{d}} \quad \text { for all } \widehat{\boldsymbol{x}} \in \mathbb{S}^{m-1} .
$$

We first study the injectivity of the modified far field operator $\mathcal{F}_{\beta}=F-F_{\beta}$. This will motivate our definition of modified interior transmission eigenvalues. To this end, we consider $g \in L^{2}\left(\mathbb{S}^{m-1}\right)$ such that $\mathcal{F}_{\beta} g=0$ in $\mathbb{S}^{m-1}$. To start the study of the modified far field operator, we can rewrite this condition by taking, in problems (2.3) and (3.1), both incident fields to be the Herglotz wave function with density $g$, that is, $p^{i}=p_{g}^{i}$ and $h^{i}=p_{g}^{i}$. Indeed, if we denote by $p_{g}^{\infty}$ and $h_{g}^{\infty}$ the far field patterns of the associated scattered waves $p_{g}^{s}$ and $h_{g}^{s}$, then $\mathcal{F}_{\beta} g=0$ implies that $p_{g}^{\infty}=h_{g}^{\infty}$ in $\mathbb{S}^{m-1}$. By Rellich's lemma, this implies that $p_{g}^{s}=h_{g}^{s}$ in $\Omega_{e}$; therefore, the transmission conditions satisfied by the displacement field $\boldsymbol{u}_{g}$ that solves (2.3) can be rewritten as

$$
\boldsymbol{T} \boldsymbol{u}_{g}=-h_{g}^{-} \boldsymbol{n}, \quad \boldsymbol{u}_{g} \cdot \boldsymbol{n}=\frac{1}{\gamma \rho_{f} \omega^{2}} \frac{\partial h_{g}^{-}}{\partial \boldsymbol{n}} \quad \text { on } \Gamma,
$$

where $h_{g}=h_{g}^{i}+h_{g}^{s}$. This discussion suggests the following definition.

Definition 1 For a fixed frequency $\omega$ and a parameter $\gamma \neq 0$, we say that $\beta \in \mathbb{C}$ is a modified interior transmission eigenvalue (mITP eigenvalue) if the following homogeneous modified interior transmission problem has a nontrivial solution $(\boldsymbol{u}, h) \in \boldsymbol{H}^{1}(\Omega) \times H^{1}(\Omega)$ : 


$$
\left.\begin{array}{ll}
\nabla \cdot \sigma(\boldsymbol{u})+\rho_{s} \omega^{2} \boldsymbol{u}=\mathbf{0} & \text { in } \Omega, \\
\frac{1}{\gamma} \Delta h+\beta k^{2} h=0 & \text { in } \Omega, \\
\boldsymbol{T} \boldsymbol{u}=-h \boldsymbol{n} & \text { on } \Gamma \\
\boldsymbol{u} \cdot \boldsymbol{n}=\frac{1}{\gamma \rho_{f} \omega^{2}} \frac{\partial h}{\partial \boldsymbol{n}} & \text { on } \Gamma .
\end{array}\right\}
$$

Notice that, by comparing Eqs. (2.4) and (3.4), it is clear that any $\beta \in \mathbb{C}$ is an mITP eigenvalue when $\omega$ is a Jones frequency (no matter the choice of $\gamma \neq 0$ ). Hence, we continue to assume that $\omega$ is not a Jones eigenvalue.

3.1 Modified transmission eigenvalues for any $\gamma \neq 0$

We next analyze the non-homogeneous counterpart of (3.4):

$$
\left.\begin{array}{ll}
\nabla \cdot \sigma(\boldsymbol{u})+\rho_{s} \omega^{2} \boldsymbol{u}=\boldsymbol{f}_{1} & \text { in } \Omega, \\
\frac{1}{\gamma} \Delta h+\beta k^{2} h=f_{2} & \text { in } \Omega, \\
\boldsymbol{u} \cdot \boldsymbol{n}-\frac{1}{\gamma \rho_{f} \omega^{2}} \frac{\partial h}{\partial \boldsymbol{n}}=g_{1} & \text { on } \Gamma, \\
\boldsymbol{T} \boldsymbol{u}+h \boldsymbol{n}=\boldsymbol{g}_{2} & \text { on } \Gamma .
\end{array}\right\}
$$

where $\boldsymbol{f}_{1} \in \boldsymbol{H}^{-1}(\Omega), f_{2} \in H^{-1}(\Omega), g_{1} \in H^{-1 / 2}(\Gamma)$ and $\boldsymbol{g}_{2} \in \boldsymbol{H}^{-1 / 2}(\Gamma)$. By reasoning in the standard way, we can formulate it in variational form as follows:

$$
\begin{aligned}
& \text { find } \boldsymbol{u} \in \boldsymbol{H}^{1}(\Omega) \text { and } h \in H^{1}(\Omega) \text { s.t. } \\
& \qquad \int_{\Omega}\left(\sigma(\boldsymbol{u}): \epsilon(\overline{\boldsymbol{v}})-\rho_{s} \omega^{2} \boldsymbol{u} \cdot \overline{\boldsymbol{v}}+\frac{1}{|\gamma|} \nabla h \cdot \nabla \bar{q}-s_{\gamma} \beta k^{2} h \bar{q}\right) \mathrm{d} \boldsymbol{x} \\
& \quad+\int_{\Gamma}\left(h \overline{\boldsymbol{v}} \cdot \boldsymbol{n}-s_{\gamma} \rho_{f} \omega^{2} \boldsymbol{u} \cdot \boldsymbol{n} \bar{q}\right) \mathrm{d} S_{\boldsymbol{x}}=-\int_{\Omega}\left(\boldsymbol{f}_{1} \cdot \overline{\boldsymbol{v}}+s_{\gamma} f_{2} \bar{q}\right) \mathrm{d} \boldsymbol{x} \\
& \quad+\int_{\Gamma}\left(\boldsymbol{g}_{2} \cdot \overline{\boldsymbol{v}}-s_{\gamma} \rho_{f} \omega^{2} g_{1} \bar{q}\right) \mathrm{dS}_{\boldsymbol{x}} \\
& \text { for all } \boldsymbol{v} \in \boldsymbol{H}^{1}(\Omega), q \in H^{1}(\Omega)
\end{aligned}
$$

where $s_{\gamma}=\gamma /|\gamma|$ denotes the sign of $\gamma$. Notice that the left-hand side defines the sesquilinear form

$$
\begin{aligned}
\tilde{a}_{\beta}((\boldsymbol{u}, h),(\boldsymbol{v}, q))= & \int_{\Omega}\left(\sigma(\boldsymbol{u}): \epsilon(\overline{\boldsymbol{v}})-\rho_{s} \omega^{2} \boldsymbol{u} \cdot \overline{\boldsymbol{v}}+\frac{1}{|\gamma|} \nabla h \cdot \nabla \bar{q}-s_{\gamma} \beta k^{2} h \bar{q}\right) \mathrm{d} \boldsymbol{x} \\
& +\int_{\Gamma}\left(h \overline{\boldsymbol{v}} \cdot \boldsymbol{n}-s_{\gamma} \rho_{f} \omega^{2} \boldsymbol{u} \cdot \boldsymbol{n} \bar{q}\right) \mathrm{d} S_{\boldsymbol{x}}
\end{aligned}
$$

which is the sum of the following two forms:

$$
\begin{aligned}
a((\boldsymbol{u}, h),(\boldsymbol{v}, q))= & \int_{\Omega}\left(\sigma(\boldsymbol{u}): \epsilon(\overline{\boldsymbol{v}})+\boldsymbol{u} \cdot \overline{\boldsymbol{v}}+\frac{1}{|\gamma|} \nabla h \cdot \nabla \bar{q}+h \bar{q}\right) \mathrm{d} \boldsymbol{x} \\
& +\int_{\Gamma}(h \overline{\boldsymbol{v}} \cdot \boldsymbol{n}-\boldsymbol{u} \cdot \boldsymbol{n} \bar{q}) \mathrm{dS}_{\boldsymbol{x}}
\end{aligned}
$$




$$
\begin{aligned}
a_{\beta}((\boldsymbol{u}, h),(\boldsymbol{v}, q))=- & \int_{\Omega}\left(\left(1+\rho_{s} \omega^{2}\right) \boldsymbol{u} \cdot \overline{\boldsymbol{v}}+\left(1+s_{\gamma} \beta k^{2}\right) h \bar{q}\right) \mathrm{d} \boldsymbol{x} \\
& +\int_{\Gamma}\left(1-s_{\gamma} \rho_{f} \omega^{2}\right) \boldsymbol{u} \cdot \boldsymbol{n} \bar{q} \mathrm{~d} S_{x} .
\end{aligned}
$$

The sesquilinear form $a(\cdot, \cdot)$ is coercive in $\left(\boldsymbol{H}^{1}(\Omega) \times H^{1}(\Omega)\right) \times\left(\boldsymbol{H}^{1}(\Omega) \times H^{1}(\Omega)\right)$. Indeed, by Korn's inequality,

$$
\begin{aligned}
\operatorname{Re}(a((\boldsymbol{v}, q),(\boldsymbol{v}, q))) & =\int_{\Omega}\left(\lambda|\nabla \cdot \boldsymbol{v}|^{2}+2 \mu|\epsilon(\boldsymbol{v})|^{2}+|\boldsymbol{v}|^{2}+\frac{1}{|\gamma|}|\nabla q|^{2}+|q|^{2}\right) \mathrm{d} \boldsymbol{x} \\
& \geq \min \{1,2 \mu\}\|\boldsymbol{v}\|_{1, \Omega}^{2}+\min \left\{1, \frac{1}{|\gamma|}\right\}\|q\|_{1, \Omega}^{2} .
\end{aligned}
$$

Therefore, since the embeddings $H^{1}(\Omega) \hookrightarrow L^{2}(\Omega)$ and $H^{1 / 2}(\Gamma) \hookrightarrow L^{2}(\Gamma)$ are compact, we can apply Fredholm theory to deduce that (3.6) is well-posed if, and only if, it admits at most one solution. We summarize this result in the following lemma.

Lemma 1 If $\beta \in \mathbb{C}$ is not an mITP eigenvalue, then the non-homogeneous modified interior transmission problem (3.5) is well-posed for data $\left(\boldsymbol{f}_{1}, f_{2}, g_{1}, \boldsymbol{g}_{2}\right)$ in $\boldsymbol{H}^{-1}(\Omega) \times H^{-1}(\Omega) \times$ $H^{-1 / 2}(\Gamma) \times \boldsymbol{H}^{-1 / 2}(\Gamma)$.

We next study mITP eigenvalues and, in particular, we analyze their location in the complex plane. To this end, notice that

$$
\operatorname{Im}\left(\tilde{a}_{\beta}\left((\boldsymbol{u}, h),\left(\boldsymbol{u},-\frac{s_{\gamma}}{\rho_{f} \omega^{2}} h\right)\right)\right)=\int_{\Omega} \frac{\operatorname{Im}(\beta) k^{2}}{\rho_{f} \omega^{2}}|h|^{2} \mathrm{~d} \boldsymbol{x} \quad \forall(\boldsymbol{u}, h) \in \boldsymbol{H}^{1}(\Omega) \times H^{1}(\Omega) .
$$

In consequence, if $\beta \in \mathbb{C} \backslash \mathbb{R}$ and $(\boldsymbol{u}, h) \in \boldsymbol{H}^{1}(\Omega) \times H^{1}(\Omega)$ solves (3.4), then $h=0$; and, under the assumption that $\omega$ is not a Jones eigenvalue, also $\boldsymbol{u}=\mathbf{0}$. Hence, mITP eigenvalues (if any) are real.

To further analyze the location of the mITP eigenvalues, we make use of a reference value $\beta_{0} \in \mathbb{C}$ which is not an mITP eigenvalue (assuming such a point exists). Then, (3.5) is well-posed for this reference value $\beta_{0}$ (see Lemma 1 ); in particular, we can define the solution operator $S_{\beta_{0}}: \boldsymbol{H}^{-1}(\Omega) \times H^{-1}(\Omega) \times H^{-1 / 2}(\Gamma) \times \boldsymbol{H}^{-1 / 2}(\Gamma) \rightarrow \boldsymbol{H}^{1}(\Omega) \times H^{1}(\Omega)$ by

$$
S_{\beta_{0}}\left(\boldsymbol{f}_{1}, f_{2}, g_{1}, \boldsymbol{g}_{2}\right)=(\boldsymbol{u}, h),
$$

where $(\boldsymbol{u}, h) \in \boldsymbol{H}^{1}(\Omega) \times H^{1}(\Omega)$ denotes the solution of (3.5) for the parameter $\beta_{0}$. By means of this operator, we characterize the mITP eigenvalues as those values $\beta \in \mathbb{C} \backslash\left\{\beta_{0}\right\}$ for which there is a non-trivial $(\boldsymbol{u}, h) \in \boldsymbol{H}^{1}(\Omega) \times H^{1}(\Omega)$ such that

$$
S_{\beta_{0}}\left(\mathbf{0},\left(\beta-\beta_{0}\right) h, 0, \mathbf{0}\right)=(\boldsymbol{u}, h) .
$$

If this is the case and $h=0$, then also $\boldsymbol{u}=\mathbf{0}$ thanks to the assumption that $\omega$ is not a Jones eigenvalue. This suggests defining the auxiliary operator $T_{\beta_{0}}: L^{2}(\Omega) \rightarrow L^{2}(\Omega)$ by $T_{\beta_{0}} f=h$ where $S_{\beta_{0}}(\mathbf{0}, f, 0, \mathbf{0})=(\boldsymbol{u}, h)$. We then characterize the mITP eigenvalues as those $\beta \in \mathbb{C}$ for which there exists a non-trivial $h \in H^{1}(\Omega)$ such that

$$
\left(\beta-\beta_{0}\right) T_{\beta_{0}} h=h ;
$$


in other words, $\beta \in \mathbb{C}$ is an mITP eigenvalue if, and only if, $\frac{1}{\beta-\beta_{0}}$ is an eigenvalue of $T_{\beta_{0}}: L^{2}(\Omega) \rightarrow L^{2}(\Omega)$.

The reasoning above leads us to study the operator $T_{\beta_{0}}: L^{2}(\Omega) \rightarrow L^{2}(\Omega)$. Notice that it is linear and bounded, and also compact (thanks to the compactness of the embedding $H^{1}(\Omega) \hookrightarrow L^{2}(\Omega)$ and the fact that $T_{\beta_{0}}: L^{2}(\Omega) \rightarrow H^{1}(\Omega)$ is linear and bounded). Also notice that its adjoint is $T_{\overline{\beta_{0}}}$ : To see this, for any $f, g \in L^{2}(\Omega)$, let us take $\left(\boldsymbol{u}_{f}, h_{f}\right)=$ $S_{\beta_{0}}(\mathbf{0}, f, 0, \mathbf{0})$ and $\left(\boldsymbol{u}_{g}, h_{g}\right)=S_{\overline{\beta_{0}}}(\mathbf{0}, g, 0, \mathbf{0})$. Using the definition of the operator $T_{\beta_{0}}$ and the equations satisfied by $h_{f}$ and $h_{g}$, we have that

$$
\begin{aligned}
\int_{\Omega} T_{\beta_{0}} f \bar{g} \mathrm{~d} \boldsymbol{x} & =\int_{\Omega} h_{f} \overline{\left(\frac{1}{\gamma} \Delta h_{g}+\overline{\beta_{0}} k^{2} h_{g}\right)} \mathrm{d} \boldsymbol{x} \\
& =\int_{\Omega}\left(-\frac{1}{\gamma} \nabla h_{f} \cdot \nabla \overline{h_{g}}+\beta_{0} k^{2} h_{f} \overline{h_{g}}\right) \mathrm{d} \boldsymbol{x}+\int_{\Gamma} \frac{1}{\gamma} h_{f} \frac{\partial \overline{h_{g}}}{\partial \boldsymbol{n}} \mathrm{dS} .
\end{aligned}
$$

In addition, the equations satisfied by $\left(\boldsymbol{u}_{f}, h_{f}\right)$ and $\left(\boldsymbol{u}_{g}, h_{g}\right)$ guarantee that, for real-valued parameters $\rho_{s}, \lambda$ and $\mu$,

$$
\begin{aligned}
0 & =\int_{\Omega}\left(-\sigma\left(\boldsymbol{u}_{f}\right): \epsilon\left(\overline{\boldsymbol{u}_{g}}\right)+\rho_{s} \omega^{2} \boldsymbol{u}_{f} \cdot \overline{\boldsymbol{u}_{g}}\right) \mathrm{d} \boldsymbol{x}+\int_{\Gamma} \boldsymbol{T} \boldsymbol{u}_{f} \cdot \overline{\boldsymbol{u}_{g}} \mathrm{~d} \mathrm{~S}_{\boldsymbol{x}} \\
& =\int_{\Omega}\left(-\sigma\left(\boldsymbol{u}_{f}\right): \epsilon\left(\overline{\boldsymbol{u}_{g}}\right)+\rho_{s} \omega^{2} \boldsymbol{u}_{f} \cdot \overline{\boldsymbol{u}_{g}}\right) \mathrm{d} \boldsymbol{x}-\int_{\Gamma} \frac{1}{\gamma \rho_{f} \omega^{2}} h_{f} \frac{\partial \overline{h_{g}}}{\partial \boldsymbol{n}} \mathrm{d} S_{\boldsymbol{x}},
\end{aligned}
$$

so that

$$
\int_{\Gamma} \frac{1}{\gamma} h_{f} \frac{\partial \overline{h_{g}}}{\partial \boldsymbol{n}} \mathrm{d} S_{\boldsymbol{x}}=\rho_{f} \omega^{2} \int_{\Omega}\left(-\sigma\left(\boldsymbol{u}_{f}\right): \epsilon\left(\overline{\boldsymbol{u}_{g}}\right)+\rho_{s} \omega^{2} \boldsymbol{u}_{f} \cdot \overline{\boldsymbol{u}_{g}}\right) \mathrm{d} \boldsymbol{x} ;
$$

similarly,

$$
\int_{\Gamma} \frac{1}{\gamma} \frac{\partial h_{f}}{\partial \boldsymbol{n}} \overline{h_{g}} \mathrm{~d} S_{\boldsymbol{x}}=\rho_{f} \omega^{2} \int_{\Omega}\left(-\sigma\left(\overline{\boldsymbol{u}_{g}}\right): \epsilon\left(\boldsymbol{u}_{f}\right)+\rho_{s} \omega^{2} \boldsymbol{u}_{f} \cdot \overline{\boldsymbol{u}_{g}}\right) \mathrm{d} \boldsymbol{x} ;
$$

and therefore

$$
\int_{\Gamma} \frac{1}{\gamma} h_{f} \frac{\partial \overline{h_{g}}}{\partial \boldsymbol{n}} \mathrm{dS}_{\boldsymbol{x}}=\int_{\Gamma} \frac{1}{\gamma} \frac{\partial h_{f}}{\partial \boldsymbol{n}} \overline{h_{g}} \mathrm{dS}_{\boldsymbol{x}} .
$$

Taking this result into (3.8), we conclude that

$$
\begin{aligned}
\int_{\Omega} T_{\beta_{0}} f \bar{g} \mathrm{~d} \boldsymbol{x} & =\int_{\Omega}\left(-\frac{1}{\gamma} \nabla h_{f} \cdot \nabla \overline{h_{g}}+\beta_{0} k^{2} h_{f} \overline{h_{g}}\right) \mathrm{d} \boldsymbol{x}+\int_{\Gamma} \frac{1}{\gamma} \frac{\partial h_{f}}{\partial \boldsymbol{n}} \overline{h_{g}} \mathrm{~d} \mathrm{~S}_{\boldsymbol{x}} \\
& =\int_{\Omega}\left(\frac{1}{\gamma} \Delta h_{f}+\beta_{0} k^{2} h_{f}\right) \overline{h_{g}} \mathrm{~d} \boldsymbol{x}=\int_{\Omega} f \overline{T_{\overline{\beta_{0}}} g} \mathrm{~d} \boldsymbol{x} .
\end{aligned}
$$

In particular, $T_{\beta_{0}}$ is self-adjoint if $\beta_{0}$ is real. Hence, if there exists at least one real number $\beta_{0}$ which is not a modified interior transmission eigenvalue, then the spectral theorem guarantees that the set of eigenvalues of $T_{\beta_{0}}$ is discrete and the only possible accumulation point is zero.

We summarize these results in the following lemma.

Lemma 2 The set of mITP eigenvalues is either the whole real line $\mathbb{R}$, or a discrete subset of $\mathbb{R}$ with no finite accumulation point (or empty).

Computational examples suggest that mITP eigenvalues exist and are discrete. 


\subsection{Modified transmission eigenvalues when $\gamma<0$}

In this subsection, we assume that $\gamma<0$ is fixed and we will see that, in particular, this hypothesis allows us to show that MITP eigenvalues exist.

First, we show that the (real) mITP eigenvalues are bounded above. To this end, let us suppose that, on the contrary, there exists a sequence of positive eigenvalues $\left\{\beta_{j}\right\}_{j \in \mathbb{N}} \subset \mathbb{R}$ that approaches $\infty$ as $j \rightarrow \infty$. We consider a sequence of associated eigenfunctions $\left\{\left(\boldsymbol{u}_{j}, h_{j}\right)\right\}_{j \in \mathbb{N}}$ normalized in $\boldsymbol{H}^{1}(\Omega) \times H^{1}(\Omega)$. Since these are eigenfunctions, it holds that $\widetilde{a}_{\beta_{j}}\left(\left(\boldsymbol{u}_{j}, h_{j}\right),\left(\boldsymbol{v}, \frac{1}{\rho_{f} \omega^{2}} q\right)\right)=0$ for all $(\boldsymbol{v}, q) \in \boldsymbol{H}^{1}(\Omega) \times H^{1}(\Omega)$; in other words,

$$
\begin{aligned}
& \int_{\Omega}\left(\sigma\left(\boldsymbol{u}_{j}\right): \epsilon(\overline{\boldsymbol{v}})-\rho_{s} \omega^{2} \boldsymbol{u}_{j} \cdot \overline{\boldsymbol{v}}-\frac{1}{\gamma \rho_{f} \omega^{2}} \nabla h_{j} \cdot \nabla \bar{q}+\frac{\beta_{j} k^{2}}{\rho_{f} \omega^{2}} h_{j} \bar{q}\right) \mathrm{d} \boldsymbol{x} \\
& \quad+\int_{\Gamma}\left(h_{j} \overline{\boldsymbol{v}} \cdot \boldsymbol{n}+\boldsymbol{u}_{j} \cdot \boldsymbol{n} \bar{q}\right) \mathrm{d} S_{\boldsymbol{x}}=0
\end{aligned}
$$

for all $(\boldsymbol{v}, q) \in \boldsymbol{H}^{1}(\Omega) \times H^{1}(\Omega)$. In particular, $\boldsymbol{u}_{j} \cdot \boldsymbol{n}=\frac{1}{\gamma \rho_{f} \omega^{2}} h_{j}$ on $\Gamma$, so that (3.9) for $(\boldsymbol{v}, q)=\left(\boldsymbol{u}_{j}, h_{j}\right)$ reads as

$$
\begin{aligned}
-\int_{\Omega} \frac{\beta_{j} k^{2}}{\rho_{f} \omega^{2}}\left|h_{j}\right|^{2} \mathrm{~d} \boldsymbol{x}= & \int_{\Omega}\left(\lambda\left|\nabla \cdot \boldsymbol{u}_{j}\right|^{2}+2 \mu\left|\epsilon\left(\boldsymbol{u}_{j}\right)\right|^{2}-\rho_{s} \omega^{2}\left|\boldsymbol{u}_{j}\right|^{2}-\frac{1}{\gamma \rho_{f} \omega^{2}}\left|\nabla h_{j}\right|^{2}\right) \mathrm{d} \boldsymbol{x} \\
& +\frac{2}{\gamma \rho_{f} \omega^{2}} \int_{\Gamma}\left|h_{j}\right|^{2} \mathrm{~d} S_{x}
\end{aligned}
$$

Notice that the right-hand side is bounded uniformly in $j \in \mathbb{N}$ (because of the normalization of the eigenfunctions $\left.\left\{\left(\boldsymbol{u}_{j}, h_{j}\right)\right\}_{j \in \mathbb{N}}\right)$, whereas $\beta_{j} \rightarrow+\infty$; thus, we deduce that $h_{j} \rightarrow 0$ in $L^{2}(\Omega)$. Let $\left\{\left(\boldsymbol{u}_{j}, h_{j}\right)\right\}_{j \in \mathbb{N}}$ be a subsequence (which for simplicity we identify with the whole sequence) that converges weakly in $\boldsymbol{H}^{1}(\Omega) \times H^{1}(\Omega)$ and strongly in $\boldsymbol{L}^{2}(\Omega) \times L^{2}(\Omega)$ to some $(\mathbf{u}, h) \in \boldsymbol{H}^{1}(\Omega) \times H^{1}(\Omega)$. Recall that we have already shown that $h=0$ in $\Omega$. Moreover, by taking the limit in (3.9) we deduce that $(\boldsymbol{u}, h) \in \boldsymbol{H}^{1}(\Omega) \times H^{1}(\Omega)$ solves (3.4) with $h=0$ in $\Omega$; in consequence, our assumption on $\omega$ not being a Jones mode guarantees that $\boldsymbol{u}=\mathbf{0}$ in $\Omega$. Summing up, $\left\{\left(\boldsymbol{u}_{j}, h_{j}\right)\right\}_{j \in \mathbb{N}}$ converges to zero weakly in $\boldsymbol{H}^{1}(\Omega) \times H^{1}(\Omega)$ and strongly in $\boldsymbol{L}^{2}(\Omega) \times L^{2}(\Omega)$. Notice that (3.10) and the strong convergence in $\boldsymbol{L}^{2}(\Omega) \times L^{2}(\Omega)$ implies that

$$
\begin{aligned}
0 & \leq \int_{\Omega}\left(\lambda\left|\nabla \cdot \boldsymbol{u}_{j}\right|^{2}+2 \mu\left|\epsilon\left(\boldsymbol{u}_{j}\right)\right|^{2}-\frac{1}{\gamma \rho_{f} \omega^{2}}\left|\nabla h_{j}\right|^{2}\right) \mathrm{d} \boldsymbol{x}+\frac{2}{\gamma \rho_{f} \omega^{2}} \int_{\Gamma}\left|h_{j}\right|^{2} \mathrm{~d} S_{\boldsymbol{x}} \\
& =\int_{\Omega}\left(\rho_{s} \omega^{2}\left|\boldsymbol{u}_{j}\right|^{2}-\frac{\beta_{j} k^{2}}{\rho_{f} \omega^{2}}\left|h_{j}\right|^{2}\right) \mathrm{d} \boldsymbol{x} \leq \int_{\Omega} \rho_{s} \omega^{2}\left|\boldsymbol{u}_{j}\right|^{2} \mathrm{~d} \boldsymbol{x} \rightarrow 0,
\end{aligned}
$$

and, in particular, $\left\|\nabla \cdot \boldsymbol{u}_{j}\right\|_{L^{2}(\Omega)} \rightarrow 0,\left\|\epsilon\left(\boldsymbol{u}_{j}\right)\right\|_{L^{2}(\Omega)^{m \times m}} \rightarrow 0$ and $\left\|\nabla h_{j}\right\|_{L^{2}(\Omega)} \rightarrow 0$. Hence, $\left\|\boldsymbol{u}_{j}\right\|_{\boldsymbol{H}^{1}(\Omega)} \rightarrow 0$ and $\left\|h_{j}\right\|_{H^{1}(\Omega)} \rightarrow 0$, which contradicts that the sequence $\left\{\left(\boldsymbol{u}_{j}, h_{j}\right)\right\}_{j \in \mathbb{N}}$ had been normalized in $\boldsymbol{H}^{1}(\Omega) \times H^{1}(\Omega)$.

Lemma 3 When $\gamma<0$, the set of mITP eigenvalues is a subset of $\mathbb{R}$ and is bounded above (or empty).

The previous result guarantees that there are real numbers that are not eigenvalues. In consequence, Lemma 2 may be modified as follows in the case when $\gamma<0$. 
Lemma 4 The set of mITP eigenvalues is either empty or a discrete subset of $\mathbb{R}$ with no finite accumulation point.

We next show that there is at least one positive mITP eigenvalue. To this end, let us denote by $\left\{\left(\boldsymbol{u}_{j}, h_{j}\right)\right\}_{j \in \mathbb{N}}$ an orthonormal basis of

$$
\mathcal{H}(\Omega)=\left\{(\boldsymbol{v}, q) \in \boldsymbol{H}^{1}(\Omega) \times H^{1}(\Omega) ; \boldsymbol{v} \cdot \boldsymbol{n}=q \text { on } \Gamma\right\}
$$

that consists of eigenfunctions associated with mITP eigenvalues $\left\{\beta_{j}\right\}_{j \in \mathbb{N}} \subset \mathbb{R}$. Notice that $\widetilde{a}_{\beta_{j}}\left(\left(\boldsymbol{u}_{j}, h_{j}\right),\left(-\boldsymbol{u}_{j}, \frac{1}{\rho_{f} \omega^{2}} h_{j}\right)\right)=0$ means

$$
\int_{\Omega} \frac{-\beta_{j} k^{2}}{\rho_{f} \omega^{2}}\left|h_{j}\right|^{2} \mathrm{~d} \boldsymbol{x}=\int_{\Omega}\left(-\lambda\left|\nabla \cdot \boldsymbol{u}_{j}\right|^{2}-2 \mu\left|\epsilon\left(\boldsymbol{u}_{j}\right)\right|^{2}+\rho_{s} \omega^{2}\left|\boldsymbol{u}_{j}\right|^{2}-\frac{1}{\gamma \rho_{f} \omega^{2}}\left|\nabla h_{j}\right|^{2}\right) \mathrm{d} \boldsymbol{x} .
$$

In particular, if all the eigenvalues were negative, we would have that

$$
0 \leq \int_{\Omega}\left(-\lambda|\nabla \cdot \boldsymbol{v}|^{2}-2 \mu|\epsilon(\boldsymbol{v})|^{2}+\rho_{s} \omega^{2}|\boldsymbol{v}|^{2}-\frac{1}{\gamma \rho_{f} \omega^{2}}|\nabla q|^{2}\right) \mathrm{d} \boldsymbol{x},
$$

for any $(\boldsymbol{v}, q) \in \mathcal{H}(\Omega)$. This leads to contradiction under suitable assumptions on the material parameters, in particular if $\lambda$, $\mu$ are large enough compared to $\rho_{s} \omega^{2}$ according to Korn's inequality: To show it, we notice that for any $\boldsymbol{v} \in \boldsymbol{H}_{0}^{1}(\Omega)$ it holds that

$$
C_{P}^{2} \min \{1,2 \mu\} \| \boldsymbol{v}||_{0, \Omega}^{2} \leq \min \{1,2 \mu\}|\boldsymbol{v}|_{1, \Omega}^{2}<\int_{\Omega}\left(\lambda|\nabla \cdot \boldsymbol{v}|^{2}+2 \mu|\epsilon(\boldsymbol{v})|^{2}\right) \mathrm{d} \boldsymbol{x},
$$

where $C_{P}>0$ is the Poincaré constant:

$$
C_{P}|| q \|_{0, \Omega} \leq|q|_{1, \Omega} \text { for all } q \in H_{0}^{1}(\Omega)
$$

therefore, if $\rho_{s} \omega^{2} \leq C_{P}^{2} \min \{1,2 \mu\}$, for any $\boldsymbol{v} \in \boldsymbol{H}_{0}^{1}(\Omega)$, we have that

$$
\int_{\Omega} \rho_{S} \omega^{2}|\boldsymbol{v}|^{2} \mathrm{~d} \boldsymbol{x}<\int_{\Omega}\left(\lambda|\nabla \cdot \boldsymbol{v}|^{2}+2 \mu|\epsilon(\boldsymbol{v})|^{2}\right) \mathrm{d} \boldsymbol{x},
$$

and then for any $\boldsymbol{v} \in \boldsymbol{H}_{0}^{1}(\Omega)$ we know that $(\boldsymbol{v}, 0) \in \mathcal{H}(\Omega)$ does not fulfill (3.11). Summarizing, we have shown the following result.

Lemma 5 Consider a solid whose material parameters $\lambda$ and $\mu$ are large enough with respect to $\rho_{s} \omega^{2}$. Then, there exists at least one positive mITP eigenvalue.

We next look for a physical meaning of the largest mITP eigenvalue. To this end, we first define $\mathcal{E}: \boldsymbol{H}^{1}(\Omega) \times H^{1}(\Omega) \rightarrow \mathbb{R}$ by

$$
\mathcal{E}(\boldsymbol{v}, q)=\int_{\Omega}\left(\sigma(\boldsymbol{v}): \epsilon(\overline{\boldsymbol{v}})-\rho_{s} \omega^{2}|\boldsymbol{v}|^{2}-\frac{1}{\gamma \rho_{f} \omega^{2}}|\nabla q|^{2}\right) \mathrm{d} \boldsymbol{x}+2 \operatorname{Re}\left(\int_{\Gamma} q \overline{\boldsymbol{v}} \cdot \boldsymbol{n} \mathrm{d} S_{x}\right) .
$$

Notice that, under suitable conditions on the material parameters $\lambda$ and $\mu$, we have that $\frac{\mathcal{E}(\boldsymbol{v}, q)}{\|q\|_{0, \Omega}^{2}}$ is bounded below for $(\boldsymbol{v}, q) \in \mathcal{H}(\Omega)$ such that $q \neq 0$. To see this, if it was not bounded, there would exist a normalized sequence $\left\{\left(\boldsymbol{v}_{j}, q_{j}\right)\right\}_{j \in \mathbb{N}} \subset \mathcal{H}(\Omega)$ such that

$$
\mathcal{E}\left(\boldsymbol{v}_{j}, q_{j}\right) \leq-j\left\|q_{j}\right\|_{0, \Omega}^{2}
$$


We emphasize that $\mathcal{E}$ is linear continuous and the eigenfunctions $\left\{\left(\boldsymbol{u}_{j}, h_{j}\right)\right\}_{j \in \mathbb{N}}$ are normalized. Therefore, $\left\{\mathcal{E}\left(\boldsymbol{v}_{j}, q_{j}\right)\right\}_{j \in \mathbb{N}}$ is bounded uniformly in $j \in \mathbb{N}$, and it follows that $\left\|q_{j}\right\|_{0, \Omega} \rightarrow 0$, that is, $q_{j} \rightarrow 0$ strongly in $L^{2}(\Omega)$. Moreover, we may take a subsequence that converges weakly in $\boldsymbol{H}^{1}(\Omega) \times H^{1}(\Omega)$ and strongly in $\boldsymbol{L}^{2}(\Omega) \times L^{2}(\Omega)$ to some $(\boldsymbol{v}, q) \in \mathcal{H}(\Omega)$; recall that we have already shown that $q=0$ in $\Omega$. Since the norm of the weak limit is not greater that the limit inferior of the norm, we have that

$$
\begin{aligned}
\int_{\Omega} \sigma(\boldsymbol{v}): \epsilon(\overline{\boldsymbol{v}}) \mathrm{d} \boldsymbol{x} & =\int_{\Omega}\left(\lambda|\nabla \cdot \boldsymbol{v}|^{2}+2 \mu|\epsilon(\boldsymbol{v})|^{2}\right) \mathrm{d} \boldsymbol{x} \\
& \leq \liminf _{j \in \int_{\mathbb{N}}} \int_{\Omega} \sigma\left(\boldsymbol{v}_{j}\right): \epsilon\left(\overline{\boldsymbol{v}_{j}}\right) \mathrm{d} \boldsymbol{x},-\frac{1}{\gamma \rho_{f} \omega^{2}} \int_{\Omega}|\nabla q|^{2} \mathrm{~d} \boldsymbol{x} \\
& \leq-\frac{1}{\gamma \rho_{f} \omega^{2}} \liminf _{j \in} \int_{\mathbb{N}}\left|\nabla q_{j}\right|^{2} \mathrm{~d} \boldsymbol{x} .
\end{aligned}
$$

The continuity of the trace implies the weak convergence of $\boldsymbol{v}_{j} \cdot \boldsymbol{n}=q_{j}$ to $\boldsymbol{v} \cdot \boldsymbol{n}=q$ in $H^{1 / 2}(\Gamma)$, so that

$$
\int_{\Gamma}|q|^{2} \mathrm{dS}_{\boldsymbol{x}} \leq \liminf _{j \in \operatorname{Nin}_{\mathbb{N}}} \int_{\Gamma}\left|q_{j}\right|^{2} \mathrm{dS} .
$$

From (3.14) rewritten as

$$
\int_{\Omega}\left(\sigma\left(\boldsymbol{v}_{j}\right): \epsilon\left(\overline{\boldsymbol{v}_{j}}\right)-\frac{1}{\gamma \rho_{f} \omega^{2}}\left|\nabla q_{j}\right|^{2}\right) \mathrm{d} \boldsymbol{x}+\frac{2}{\gamma \rho_{f} \omega^{2}} \int_{\Gamma}\left|q_{j}\right|^{2} \mathrm{~d} S_{\boldsymbol{x}} \leq \int_{\Omega} \rho_{s} \omega^{2}\left|\boldsymbol{v}_{j}\right|^{2} \mathrm{~d} \boldsymbol{x}-j|| q_{j} \|_{0, \Omega}^{2},
$$

and the fact that, because of the strong convergence of $\boldsymbol{v}_{j}$ toward $\boldsymbol{v}$ in $\boldsymbol{L}^{2}(\Omega)$,

$$
\liminf _{j \in \mathbb{N}} \int_{\Omega} \rho_{s} \omega^{2}\left|\boldsymbol{v}_{j}\right|^{2} \mathrm{~d} \boldsymbol{x}=\lim _{j \in \mathbb{N}} \int_{\Omega} \rho_{s} \omega^{2}\left|\boldsymbol{v}_{j}\right|^{2} \mathrm{~d} \boldsymbol{x}=\rho_{s} \omega^{2}\|\boldsymbol{v}\|_{0, \Omega}^{2},
$$

we have that

$$
\liminf _{j \in \in}\left(\int_{\Omega}\left(\sigma\left(\boldsymbol{v}_{j}\right): \epsilon\left(\overline{\boldsymbol{v}_{j}}\right)-\frac{1}{\gamma \rho_{f} \omega^{2}}\left|\nabla q_{j}\right|^{2}\right) \mathrm{d} \boldsymbol{x}+\frac{2}{\gamma \rho_{f} \omega^{2}} \int_{\Gamma}\left|q_{j}\right|^{2} \mathrm{~d} S_{\boldsymbol{x}}\right) \leq \rho_{s} \omega^{2}\|\boldsymbol{v}\|_{0, \Omega}^{2} .
$$

Therefore,

$$
\int_{\Omega}\left(\sigma(\boldsymbol{v}): \epsilon(\overline{\boldsymbol{v}})-\frac{1}{\gamma \rho_{f} \omega^{2}}|\nabla q|^{2}\right) \mathrm{d} \boldsymbol{x}+\frac{2}{\gamma \rho_{f} \omega^{2}} \int_{\Gamma}|q|^{2} \mathrm{~d} S_{x} \leq \rho_{s} \omega^{2}\|\boldsymbol{v}\|_{0, \Omega}^{2} .
$$

In particular,

$$
\int_{\Omega}\left(\lambda|\nabla \cdot \boldsymbol{v}|^{2}+2 \mu|\epsilon(\boldsymbol{v})|^{2}\right) \mathrm{d} \boldsymbol{x}+\frac{2}{\gamma \rho_{f} \omega^{2}} \int_{\Gamma}|\boldsymbol{v} \cdot \boldsymbol{n}|^{2} \mathrm{~d} S_{x} \leq \rho_{s} \omega^{2}\|\boldsymbol{v}\|_{0, \Omega}^{2} .
$$

This leads to a contradiction if $\lambda$ and $\mu$ are large enough compared to $\rho_{s} \omega^{2}$, see (3.13) above.

Summing up, we have just shown that, for large enough $\lambda$ and $\mu$, there exists

$$
\Lambda=\inf _{(\boldsymbol{v}, q) \in \mathcal{H}(\Omega), q \neq 0} \frac{\mathcal{E}(\boldsymbol{v}, q)}{\|q\|_{0, \Omega}^{2}} .
$$


We next look for an estimate for the largest mITP eigenvalue $\beta_{\max }$ by using the above defined $\Lambda$. To this end, we start by considering any mITP eigenvalue $\beta$ and denote by $(\boldsymbol{u}, h) \in \mathcal{H}(\Omega)$ an associated eigenfunction of its. As in (3.10), from the fact that it is an eigenfunction we have that

$$
\begin{aligned}
-\int_{\Omega} \frac{\beta k^{2}}{\rho_{f} \omega^{2}}|h|^{2} \mathrm{~d} \boldsymbol{x}=\int_{\Omega} & \left(\lambda|\nabla \cdot \boldsymbol{u}|^{2}+2 \mu|\epsilon(\boldsymbol{u})|^{2}-\rho_{s} \omega^{2}|\boldsymbol{u}|^{2}-\frac{1}{\gamma \rho_{f} \omega^{2}}|\nabla h|^{2}\right) \mathrm{d} \boldsymbol{x} \\
& +\frac{2}{\gamma \rho_{f} \omega^{2}} \int_{\Gamma}|h|^{2} \mathrm{dS}_{\boldsymbol{x}} .
\end{aligned}
$$

This is equivalently rewritten using the definition of $\mathcal{E}$ and the transmission conditions as $-\frac{\beta k^{2}}{\rho_{f} \omega^{2}}\|h\|_{0, \Omega}^{2}=\mathcal{E}(\boldsymbol{u}, h)$. Also notice that $h \neq 0$ because, otherwise, $\boldsymbol{u}$ would also cancel under the assumption that $\omega$ is not a Jones mode. Therefore, $-\beta \geq \frac{\rho_{f} \omega^{2}}{k^{2}} \Lambda$, or equivalently, in terms of the largest mITP eigenvalue $\beta_{\max }$, we have

$$
\beta_{\max } \leq-\frac{\rho_{f} \omega^{2}}{k^{2}} \Lambda
$$

We next show that this bound is indeed optimal. To this end, let us reason as usual in the derivation of the Courant min-max principle (cf. [3, Th. 6.13] and references therein): We consider $\left\{\left(\boldsymbol{u}_{j}, h_{j}\right)\right\}_{j \in \mathbb{N}}$ an orthonormal basis of $\mathcal{H}(\Omega)$ that consists of eigenvectors associated with mITP eigenvalues $\left\{\beta_{j}\right\}_{j \in \mathbb{N}} \subset \mathbb{R}$. We rewrite $\widetilde{a}_{\beta_{j}}\left(\left(\boldsymbol{u}_{j}, h_{j}\right),(\boldsymbol{v}, q)\right)=0$ as

$$
-A\left(\left(\boldsymbol{u}_{j}, h_{j}\right),(\boldsymbol{v}, q)\right)=\int_{\Omega} \frac{\beta_{j} k^{2}}{\rho_{f} \omega^{2}} h \bar{q} \mathrm{~d} \boldsymbol{x},
$$

where

$$
\begin{aligned}
A((\boldsymbol{u}, h),(\boldsymbol{v}, q))=\int_{\Omega} & \left(-\sigma(\boldsymbol{u}): \epsilon(\overline{\boldsymbol{v}})+\rho_{s} \omega^{2} \boldsymbol{u} \cdot \overline{\boldsymbol{v}}\right. \\
& \left.+\frac{1}{\gamma \rho_{f} \omega^{2}} \nabla h \cdot \nabla \bar{q}\right) \mathrm{d} \boldsymbol{x}-2 \operatorname{Re}\left(\int_{\Gamma} h \bar{q} \mathrm{~d} S_{x}\right) .
\end{aligned}
$$

Since $\left\{\left(\boldsymbol{u}_{j}, h_{j}\right)\right\}_{j \in \mathbb{N}}$ is an orthonormal basis of $\mathcal{H}(\Omega)$, we deduce that for any $(\boldsymbol{v}, q)=$ $\sum_{j \in \mathbb{N}} a_{j}\left(\boldsymbol{u}_{j}, h_{j}\right) \in \mathcal{H}(\Omega)$ it holds

$$
-A((\boldsymbol{v}, q),(\boldsymbol{v}, q))=\sum_{j \in \mathbb{N}} \frac{\beta_{j} k^{2}}{\rho_{f} \omega^{2}}\left|a_{j}\right|^{2}\left\|h_{j}\right\|_{L^{2}(\Omega)}^{2} .
$$

Hence,

$$
-\Lambda=\sup _{(\boldsymbol{v}, q) \in \mathcal{H}(\Omega), q \neq 0} \frac{-A((\boldsymbol{v}, q),(\boldsymbol{v}, q))}{\|q\|_{L^{2}(\Omega)}^{2}}=\frac{\beta_{\max } k^{2}}{\rho_{f} \omega^{2}} .
$$

We state this result in the following lemma.

Lemma 6 Suppose that the material parameters $\lambda$ and $\mu$ satisfy (2.1) and $\mu$ is large enough with respect to $\rho_{s} \omega^{2}$, that is, $\rho_{s} \omega^{2} \leq C_{P}^{2} \min \{1,2 \mu\}$. Then, the largest mITP eigenvalue is $\beta_{\max }=-\frac{\rho_{f} \omega^{2}}{k^{2}} \Lambda$. 


\section{Determination of modified interior transmission eigenvalues from far field data}

Let us consider again the general situation $\gamma \neq 0$ (positive or negative). The aim of this section is to show that we can approximate the mITP eigenvalues from far field data by solving approximately several modified far field equations for some points inside the target. For this strategy to make sense, we need $\mathcal{F}_{\beta}: L^{2}\left(\mathbb{S}^{m-1}\right) \rightarrow L^{2}\left(\mathbb{S}^{m-1}\right)$ to be injective and to have dense range. We can repeat the usual reasoning (cf. $[2,8]$ ) to deduce that $\mathcal{F}_{\beta}$ is injective and has dense range in $L^{2}\left(\mathbb{S}^{m-1}\right)$ as long as $\beta$ is not an mITP eigenvalue whose eigenmode has the form $\left(\boldsymbol{u}_{g}, h_{g}\right)$ for some $g \in L^{2}\left(\mathbb{S}^{m-1}\right)$.

Let $\Phi_{z}$ denote the fundamental solution of the Helmholtz equation with source point at $z$ so that, for $x \neq z$,

$$
\Phi_{\boldsymbol{z}}(\boldsymbol{x})= \begin{cases}\frac{i}{4} H_{0}^{(1)}\left(k_{f}|\boldsymbol{x}-\boldsymbol{z}|\right) & \text { if } m=2, \\ \frac{1}{4 \pi} \frac{\exp \left(i k_{f}|\boldsymbol{x}-\boldsymbol{z}|\right)}{|\boldsymbol{x}-\boldsymbol{z}|} & \text { if } m=3 .\end{cases}
$$

Since $\Phi_{z}$ is a radiating solution of the Helmholtz equation, it has a far field pattern, and this is given by:

$$
\Phi_{\mathbf{z}}^{\infty}(\widehat{\mathbf{x}})=\frac{\exp (i \pi / 4)}{\sqrt{8 \pi k}} \exp \left(-i k_{f} \widehat{\mathbf{x}} \cdot \mathbf{z}\right), \quad \widehat{\mathbf{x}} \in \mathbb{S}^{m-1} .
$$

The algorithm for identifying mITP eigenvalues is based on solving approximately a discrete version of the modified far field equation

$$
\mathcal{F}_{\beta} g_{\mathbf{z}}=\Phi_{\mathbf{z}}^{\infty}
$$

where $\mathbf{z} \in \Omega$ is an auxiliary point chosen as part of the algorithm. Next, we examine the role of the parameter $\beta$ in the behavior of (approximate) solutions $g_{z} \in L^{2}\left(\mathbb{S}^{m-1}\right)$ of the modified far field equations (4.1) for points $z$ inside the target $\Omega$.

\subsection{Behavior at values of $\beta$ which are not mITP eigenvalues}

Let us consider any point $z \in \Omega$ and then seek $g \in L^{2}\left(\mathbb{S}^{m-1}\right)$ that solves approximately

$$
\mathcal{F}_{\beta} g=p_{g}^{\infty}-h_{g}^{\infty}=\Phi_{z}^{\infty} \text { a.e. in } \mathbb{S}^{m-1} .
$$

By Rellich's lemma, this happens if, and only if,

$$
p_{g}^{s}-h_{g}^{s}=\Phi_{z} \quad \text { a.e. in } \Omega_{e} ;
$$

in particular, $p_{g}^{+}-h_{g}^{+}=\Phi_{z}$ and $\frac{\partial p_{g}^{+}}{\partial \boldsymbol{n}}-\frac{\partial h_{g}^{+}}{\partial \boldsymbol{n}}=\frac{\partial \Phi_{z}}{\partial \boldsymbol{n}}$ on $\Gamma$. Thanks to the transmission conditions for the fields $\boldsymbol{u}_{g}$ and $p_{g}$ in (2.3), together with those for $h_{g}$ in (3.1),

$$
\boldsymbol{u}_{g} \cdot \boldsymbol{n}-\frac{1}{\gamma \rho_{f} \omega^{2}} \frac{\partial h_{g}^{-}}{\partial \boldsymbol{n}}=\frac{1}{\rho_{f} \omega^{2}} \frac{\partial \Phi_{z}}{\partial \boldsymbol{n}} \quad \text { and } \quad \boldsymbol{t}\left(\boldsymbol{u}_{g}\right)=-h_{g}^{-} \boldsymbol{n}-\Phi_{z} \boldsymbol{n} \quad \text { on } \Gamma .
$$

Thus, in the domain $\Omega$ we are looking for $\boldsymbol{u}=\boldsymbol{u}_{g} \in \boldsymbol{H}^{1}(\Omega)$ and $h^{-}=h_{g}^{-} \in H^{1}(\Omega)$ such that

$$
\left.\begin{array}{ll}
\nabla \cdot \sigma(\boldsymbol{u})+\rho_{s} \omega^{2} \boldsymbol{u}=\mathbf{0} & \text { in } \Omega, \\
\frac{1}{\gamma} \Delta h^{-}+\beta k^{2} h^{-}=0 & \text { in } \Omega, \\
\boldsymbol{u} \cdot \boldsymbol{n}-\frac{1}{\gamma \rho_{f} \omega^{2}} \frac{\partial h^{-}}{\partial \boldsymbol{n}}=\frac{1}{\rho_{f} \omega^{2}} \frac{\partial \Phi_{z}}{\partial \boldsymbol{n}} & \text { on } \Gamma, \\
\boldsymbol{t}(\boldsymbol{u})+h^{-} \boldsymbol{n}=-\Phi_{z} \boldsymbol{n} & \text { on } \Gamma .
\end{array}\right\}
$$


Assuming that $\beta$ is not an mITP eigenvalue, the problem above is well-posed and has a unique solution $\left(\boldsymbol{u}, h^{-}\right) \in H^{1}(\Omega)^{n} \times H^{1}(\Omega)$, see Lemma 1 ; however, the unique solution of this problem is not necessarily of the form $\boldsymbol{u}=\boldsymbol{u}_{g}$ and $h^{-}=h_{g}^{-}$with $g \in L^{2}\left(\mathbb{S}^{m-1}\right)$. Furthermore, in the exterior domain $\Omega_{e}$, we are looking for a suitable function $g \in L^{2}\left(\mathbb{S}^{n-1}\right)$ to build $p=p_{g} \in H^{1}\left(\Omega_{e}\right)$ and $h^{+}=h_{g}^{+} \in H_{\mathrm{loc}}^{1}\left(\Omega_{e}\right)$. To this end, we take $p=h^{+}+\Phi_{z}$ in $\Omega_{e}$, where $h^{+}$solves

$$
\begin{aligned}
& \Delta h^{+}+k^{2} h^{+}=0 \quad \text { in } \Omega_{e}, \\
& h^{+}=h^{-} \quad \text { on } \Gamma \text {, } \\
& \frac{\partial h^{+}}{\partial \boldsymbol{n}}=\frac{1}{\gamma} \frac{\partial h^{-}}{\partial \boldsymbol{n}} \quad \text { on } \Gamma \text {, } \\
& h^{+}=h^{i}+h^{+, s} \text { and } \quad \frac{\partial h^{+, s}}{\partial r}-i k h^{+, s}=\mathrm{O}\left(r^{-(m-1) / 2}\right) \text { as } r \rightarrow \infty .
\end{aligned}
$$

This problem seems to have too many constraints, but we emphasize that the incident field $h^{i}$ is not given, but we choose both $h^{i}$ and $h^{+, s}$. In particular, for the incident field $h^{i}$, let us not require it to be a Herglotz function and allow it to be just an admissible incident field, that is, $h^{i} \in H^{1}(\Omega)$ such that $\Delta h^{i}+k^{2} h^{i}=0$ in $\Omega$. Accordingly, (4.3) consists of finding $h^{i} \in H^{1}(\Omega)$ and $h^{+, s} \in H_{\text {loc }}^{1}\left(\Omega_{e}\right)$ such that

$$
\left.\begin{array}{ll}
\Delta h^{i}+k^{2} h^{i}=0 & \text { in } \Omega, \\
\Delta h^{+, s}+k^{2} h^{+, s}=0 & \text { in } \Omega_{e}, \\
h^{i}+h^{+, s}=h^{-} & \text {on } \Gamma, \\
\frac{\partial h^{i}}{\partial \boldsymbol{n}}+\frac{\partial h^{s,+}}{\partial \boldsymbol{n}}=\frac{1}{\gamma} \frac{\partial h^{-}}{\partial \boldsymbol{n}} & \text { on } \Gamma, \\
\frac{\partial h^{+, s}}{\partial r}-i k h^{+, s}=\mathrm{O}\left(r^{-(m-1) / 2}\right) & \text { as } r \rightarrow \infty
\end{array}\right\}
$$

This problem is a well-posed transmission problem, although once again we emphasize that its unique solution is not necessarily of the form $h^{i}=h_{g}^{i}$ and $h^{+, s}=h_{g}^{+, s}$ with $g \in L^{2}\left(\mathbb{S}^{m-1}\right)$.

Summing up, let us define the space of generalized incident fields by

$$
\mathbb{H}_{\text {inc }}(\Omega)=\left\{q \in H^{1}(\Omega) ; \Delta q+k^{2} q=0 \text { in } \Omega\right\} .
$$

Then, we have seen that, for any $z \in \Omega$ and when $\beta \in \mathbb{C}$ is not an mITP eigenvalue, there exists some $p^{i}=h^{i} \in \mathbb{H}_{\text {inc }}(\Omega)$ for which the far field patterns of the associated scattered fields that solve (2.3) and (3.1) satisfy

$$
p^{\infty}-h^{\infty}=\Phi_{z}^{\infty} .
$$

We can formalize this behavior in terms of the far field operator $\mathcal{F}_{\beta}$ by an appropriate factorization. More precisely, we consider the well-defined and bounded operator $\mathcal{G}$ : $\mathbb{H}_{\text {inc }}(\Omega) \rightarrow L^{2}\left(\mathbb{S}^{m-1}\right)$, where $\mathcal{G} p^{i}=p^{\infty}$ represents the far field pattern of the scattered wave that solves (2.3) for the incident field $p^{i}$. Similarly, $\mathcal{G}_{\beta}: \mathbb{H}_{\text {inc }}(\Omega) \rightarrow L^{2}\left(\mathbb{S}^{m-1}\right)$, where $\mathcal{G}_{\beta} h^{i}=h^{\infty}$ represents the far field pattern of the scattered wave that solves (3.1) for the incident field $h^{i}$. In addition, we define $\mathcal{H}: L^{2}\left(\mathbb{S}^{m-1}\right) \rightarrow \mathbb{H}_{\text {inc }}(\Omega)$ that maps each function $g$ into the associated Herglotz wave function $\mathcal{H} g=p_{g}^{i}$ with kernel $g$. Then, $\mathcal{F}_{\beta}=\left(\mathcal{G}-\mathcal{G}_{\beta}\right) \circ \mathcal{H}: L^{2}\left(\mathbb{S}^{m-1}\right) \rightarrow \mathbb{H}_{i n c}(\Omega) \rightarrow L^{2}\left(\mathbb{S}^{m-1}\right)$. With this notation, what we have just shown is that $\Phi_{z}^{\infty}$ is in the range of $\left(\mathcal{G}-\mathcal{G}_{\beta}\right)$. 


\subsection{Behavior at values of $\beta$ which are mITP eigenvalues}

If $\beta \in \mathbb{R}$ is an mITP eigenvalue, by Definition 1 we know that there exists $\left(\boldsymbol{u}_{\beta}, h_{\beta}\right) \in$ $\boldsymbol{H}^{1}(\Omega) \times H^{1}(\Omega)$ that is a non-trivial solution of

$$
\left.\begin{array}{ll}
\nabla \cdot \sigma\left(\boldsymbol{u}_{\beta}\right)+\rho_{s} \omega^{2} \boldsymbol{u}_{\beta}=\mathbf{0} & \text { in } \Omega, \\
\frac{1}{\gamma} \Delta h_{\beta}+\beta k^{2} h_{\beta}=0 & \text { in } \Omega, \\
\boldsymbol{u}_{\beta} \cdot \boldsymbol{n}=\frac{1}{\gamma \rho_{f} \omega^{2}} \frac{\partial h_{\beta}}{\partial \boldsymbol{n}} & \text { on } \Gamma, \\
\boldsymbol{t}\left(\boldsymbol{u}_{\beta}\right)=-h_{\beta} \boldsymbol{n} & \text { on } \Gamma .
\end{array}\right\}
$$

Notice that, by defining $h_{\beta}^{-}=h_{\beta}$ in $\Omega$ and reasoning as for the previous case (see comments below (4.3)), we know that there also exist $h_{\beta}^{i} \in \mathbb{H}_{\text {inc }}(\Omega)$ and $h_{\beta}^{+} \in H_{\mathrm{loc}}^{1}\left(\Omega_{e}\right)$ such that

$$
\left.\begin{array}{ll}
\Delta h_{\beta}^{+}+k^{2} h_{\beta}^{+}=0 & \text { in } \Omega_{e}, \\
h_{\beta}^{+}=h_{\beta}^{-} & \text {on } \Gamma, \\
\frac{\partial h_{\beta}^{+}}{\partial \boldsymbol{n}}=\frac{1}{\gamma} \frac{\partial h_{\beta}^{-}}{\partial \boldsymbol{n}} & \text { on } \Gamma, \\
h_{\beta}^{+}=h_{\beta}^{i}+h_{\beta}^{+, s} & \text { and } \quad \frac{\partial h_{\beta}^{+, s}}{\partial r}-i k h_{\beta}^{+, s}=\mathrm{O}\left(r^{-(n-1) / 2}\right) \text { as } r \rightarrow \infty .
\end{array}\right\}
$$

We then take $p_{\beta}^{i}=h_{\beta}^{i} \in \mathbb{H}_{\text {inc }}(\Omega)$ and $p_{\beta}=h_{\beta}^{+}$in $\Omega_{e}$, and notice that $\left(\boldsymbol{u}_{\beta}, p_{\beta}\right) \in$ $\boldsymbol{H}^{1}(\Omega) \times H_{\mathrm{loc}}^{1}\left(\Omega_{e}\right)$ solves (2.3) and $h_{\beta} \in H_{\mathrm{loc}}^{1}\left(\Omega \cup \Omega_{e}\right)$ satisfies (3.1).

Let us assume that for a given point $z \in \Omega$ there exists $p^{i} \in \mathbb{H}_{\text {inc }}(\Omega)$ such that $(\mathcal{G}-$ $\left.\mathcal{G}_{\beta}\right) p^{i}=\Phi_{z}^{\infty}$. Reasoning as for the previous case, we can deduce that the associated fields $\boldsymbol{u} \in \boldsymbol{H}^{1}(\Omega)$ and $h^{-} \in H^{1}(\Omega)$ satisfy the equations of (4.2). Therefore, by combining the variational formulation (3.6) for $\boldsymbol{v}=\boldsymbol{u}_{\beta}$ and $q=h_{\beta}$ (associated with null data functions $\boldsymbol{f}_{1}, f_{2}, g_{1}$ and $\boldsymbol{g}_{2}$ ), with that for $\boldsymbol{v}=\boldsymbol{u}$ and $q=h^{-}$(that corresponds to the data functions $\boldsymbol{f}_{1}=\mathbf{0}, f_{2}=0, g_{1}=\frac{1}{\rho_{f} \omega^{2}} \frac{\partial \Phi_{z}}{\partial \boldsymbol{n}}$ and $\left.\boldsymbol{g}_{2}=-\Phi_{z} \boldsymbol{n}\right)$, we have that

$$
\int_{\Gamma}\left(\Phi_{z} \overline{\boldsymbol{u}_{\beta}} \cdot \boldsymbol{n}-\frac{1}{\gamma \rho_{f} \omega^{2}} \frac{\partial \Phi_{z}}{\partial \boldsymbol{n}} \overline{h_{\beta}}\right) \mathrm{d} S_{\boldsymbol{x}}=0 .
$$

Using the transmission conditions on $\Gamma$ satisfied by $\left(\boldsymbol{u}_{\beta}, h_{\beta}\right)$, this is equivalent to

$$
\int_{\Gamma}\left(\Phi_{z} \frac{\partial \overline{h_{\beta}}}{\partial \boldsymbol{n}}-\frac{\partial \Phi_{z}}{\partial \boldsymbol{n}} \overline{h_{\beta}}\right) \mathrm{d} S_{\boldsymbol{x}}=0 .
$$

The left-hand side of this equation is the integral representation of $\overline{h_{\beta}}$ for points $z$ inside $\Omega$. Therefore, if this condition is fulfilled for a subset of $\Omega$ with nonzero measure, then $\overline{h_{\beta}}$ vanishes in such domain and, in consequence, also in the whole domain $\Omega$. Under the assumption on $\omega$ not being a Jones frequency, it follows that also $\boldsymbol{u}_{\beta}=\mathbf{0}$ vanishes in $\Omega$. This contradicts that $\left(\boldsymbol{u}_{\beta}, h_{\beta}\right)$ is a non-trivial mode associated with the modified interior transmission eigenvalue $\beta$.

Summarizing, we have shown the following theorem.

Theorem 1 If $\beta \in \mathbb{C}$ is not an mITP eigenvalue, then for almost any $z \in \Omega$, it holds that $\Phi_{z}^{\infty}$ is in the range of $\left(\mathcal{G}-\mathcal{G}_{\beta}\right)$. On the contrary, if $\beta \in \mathbb{C}$ is an mITP eigenvalue and $B \subset \Omega$ has nonzero measure, then it is not possible that $\Phi_{z}^{\infty}$ is in the range of $\left(\mathcal{G}-\mathcal{G}_{\beta}\right)$ for a.e. $z \in B$. 


\section{Numerical examples}

The numerical experiments we will now provide give preliminary evidence that some target signatures may be computed from the far field data. For a given experiment, there is a limit to the number of mITP eigenvalues we can detect from noisy data. Our results demonstrate the sensitivity of the detected MITP eigenvalues to some of the material parameters of the solid.

The general outline of the numerical tests is as follows:

1. We choose a scatterer (in our tests, the unit disk or unit square), and the fluid and solid properties.

2. We solve the forward problem using a coupled finite element and spectral boundary element method [22]. The same code is used to compute the solution of the auxiliary scattering problem for a discrete set of $\beta$ in the interval where we seek mITP eigenvalues. These codes produce a discrete approximation to the far fields of each problem at equally spaced points on $\mathbb{S}^{1}$.

3. In order to detect the family of mITP eigenvalues from the above far field data, the modified far field equation (4.1) is discretized using the trapezoidal rule with nodes at the far field data points. The discretized version of equation (4.1) is solved by Tikhonov regularization for several choices of auxiliary source points $\mathbf{z}$ located (randomly) in an open region that is known a priori to lie within the target.

4. We plot the average (averaged over the points $\mathbf{z}$ ) of the $L^{2}$-norm of $g_{\mathbf{z}}$ against $\beta$. Theorem 1 suggests that peaks in this graph should indicate the presence of an mITP eigenvalue.

5. To compare the peaks in the graphs against the mITP eigenvalues, we compute these eigenvalues by solving the eigenvalue problem (3.4) with a straightforward eigenvalue code developed in Netgen [28].

Next, we give some more details about the numerical tests provided here: We first consider the unit disk (centered at the origin and with unit radius) and the parameters $\omega=2$ and $\rho_{f}=\rho_{s}=1$; for this case, we choose the points $\mathbf{z} \in D$ to be 20 randomly located points in $[-0.5,0.5]^{2}$. To investigate non-smooth geometries, we also consider the unit square $[-0.5,0.5]^{2}$ and then take the same parameters except the wavenumber; indeed, in this situation, we choose $\omega=1$ (in order to keep the same ratio between the target size and the wavelength) and we relocate the 20 random points under study to $[-0.25,0.25]^{2}$ (so they lie inside the target). We take 64 incident plane waves, whose angles of incidence are $\{2 j \pi / 64 ; j=0,1, \ldots, 63\}$. We compute the far field data associated with each incident wave by a combination of spectral BEM and FEM (cf. [22]), and add pointwise $2 \%$ uniformly distributed noise to the synthetic data (to help avoid inverse crimes). We finally solve the equation (4.1) using Tikhonov regularization with the small fixed regularization parameter $10^{-3}$.

\subsection{Numerical results for $\gamma>0$}

In Fig. 1, we compare the mITP eigenvalues recovered by the steps describe above against those computed by solving (3.4) using our Netgen code. The parameter $\gamma$ is taken to be 2 both for the unit circle and the unit square, and we choose $\mu=0.5$ and $\lambda=1$. In each case at least four eigenvalues are detected, although some of these are slightly displaced 

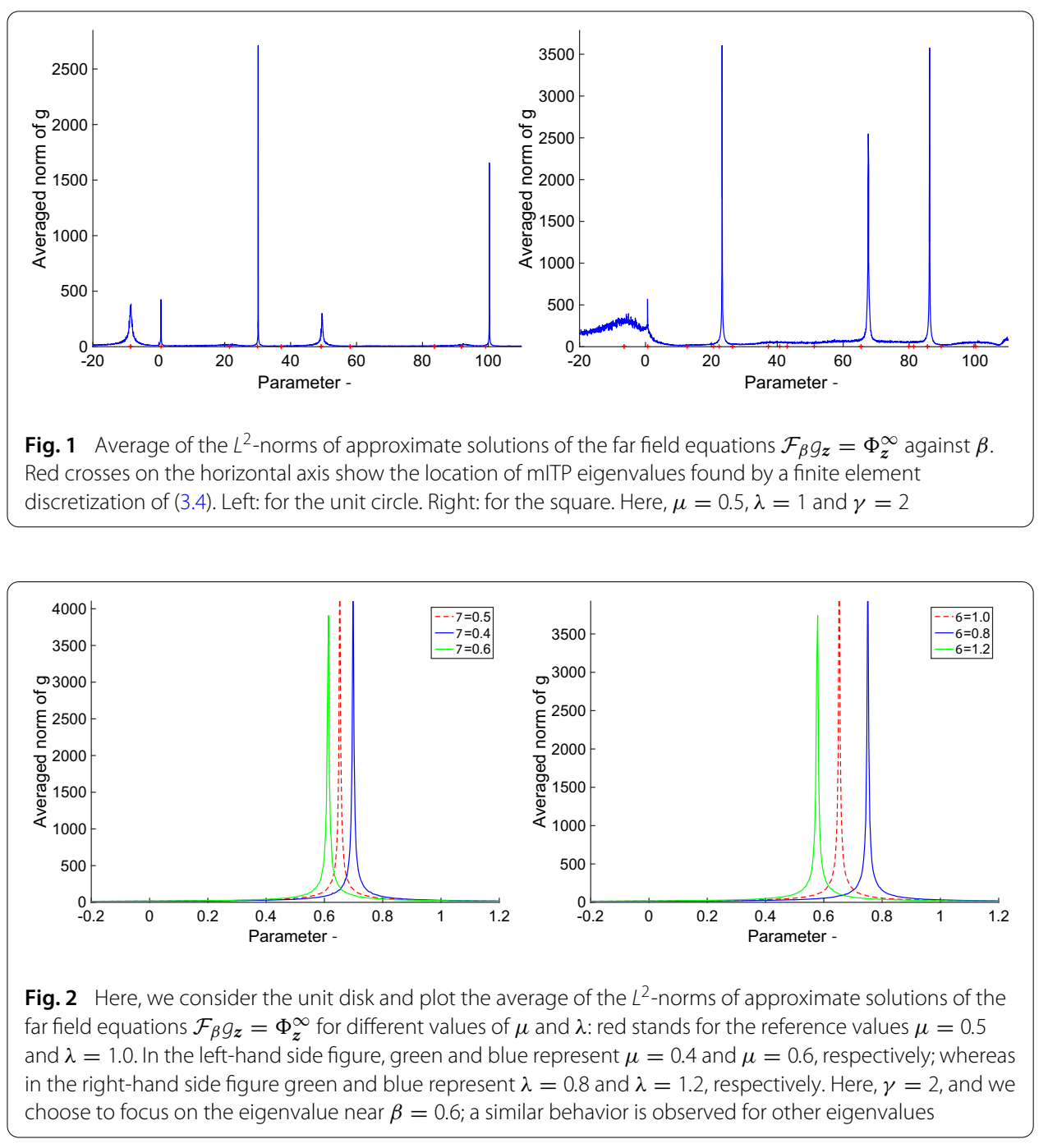

compared to the Netgen eigenvalue (specially when they are large and in the case of the square).

We next investigate the sensitivity of mITP eigenvalues with respect to changes in the elasticity parameters. More precisely, in Figs. 2 and 3 we show the behavior of the solutions of the far field equations for the circle and the square for different values of $\mu$ and $\lambda$, respectively. It is clear that mITP eigenvalues are sensitive to changes in the parameters. It is not known if the monotonic dependence shown in these examples is a general characteristic of mITP eigenvalues.

\subsection{Numerical results for $\gamma<0$}

Now, the parameter $\gamma$ is taken to be -2 both for the unit circle and the unit square. In Fig. 4, we compare the recovered mITP eigenvalues against those computed by solving (3.4) with our Netgen code. In each case at least four eigenvalues are detected, although some of these are again slightly displaced compared to the Netgen eigenvalue.

As for the case $\gamma>0$, we also investigate the sensitivity of mITP eigenvalues with respect to changes in the elasticity parameters: In Figs. 5 and 6, we show the behavior of 

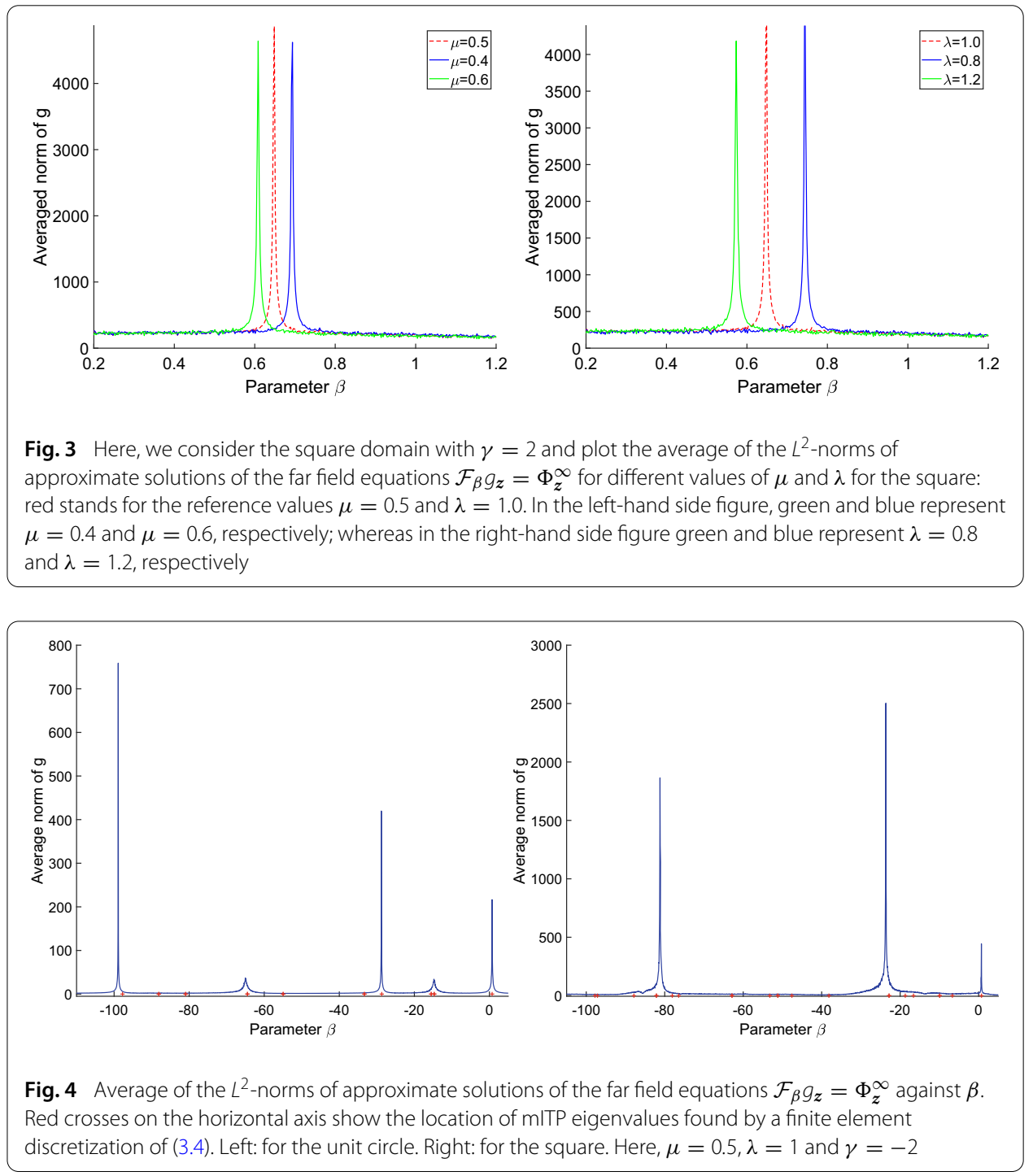

the solutions of the far field equations for the circle and the square for different values of $\mu$ and $\lambda$, respectively. As happened for the opposite sign case, the mITP eigenvalues are sensitive to changes in the parameters.

\section{Conclusion}

We have shown that mITP eigenvalues can be detected from far field data. One issue remaining is that we have not verified the existence of mITP eigenvalues when $\gamma>0$, although they have been observed by directly solving the eigenvalue problem, and from the behavior of the modified far field equation. For $\gamma<0$, the theory is more complete, and eigenvalues are known to exist. Since the case $\gamma<0$ also shows sensitivity to changes in bulk $\lambda$ and $\mu$, it seems that this case is appropriate for further investigation.

We remark that the natural and metamaterial cases studied here are by no means the only possible modifications for introducing a reference medium. For example, we could try to use a fictitious solid in $\Omega$ with a parameter in the lower order term (similar to $\beta$ in our natural and metamaterial cases) that can be used to generate an eigenvalue problem. In this paper, we chose to use the Helmholtz equation in $\Omega$ to avoid having to solve auxiliary 

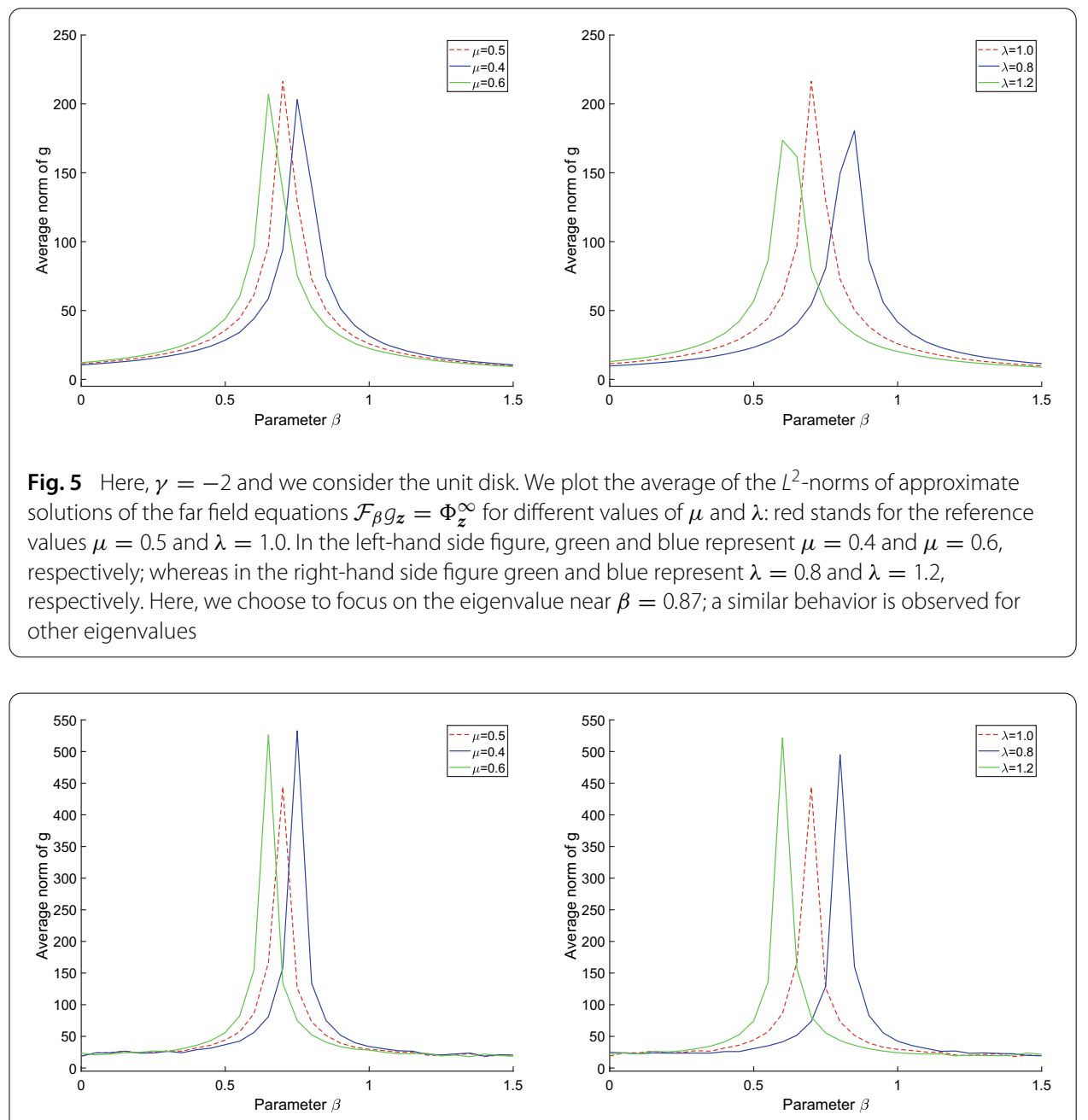

Fig. 6 Here, $\gamma=-2$ and we consider the square domain. We plot the average of the $L^{2}$-norms of approximate solutions of the far field equations $\mathcal{F}_{\beta} g_{z}=\Phi_{z}^{\infty}$ for different values of $\mu$ and $\lambda$ : red stands for the reference values $\mu=0.5$ and $\lambda=1.0$. In the left-hand side figure, green and blue represent $\mu=0.4$ and $\mu=0.6$, respectively; whereas in the right-hand side figure green and blue represent $\lambda=0.8$ and $\lambda=1.2$, respectively. The zoom is made on the eigenvalue near $\beta=0.7$; a similar behavior is observed in other eigenvalues

problems involving the time harmonic elastic wave equation. But this is more a matter of convenience than necessity, and the use of an elastic reference medium will be the object of a future investigation.

Acknowledgements

The research of P.M. is partially supported by the US Air Force Office of Scientific Research (AFOSR) under grant number FA9550-20-1-0024. The visit of V.S. in the University of Delaware was funded by the program Movilidades de Excelencia of the University of Oviedo, and her research is partially supported by the project MTM2017-87162-P of MINECO.

\section{Funding}

Peter Monk Partially supported by the US Air Force Office of Scientific Research (AFOSR) under Grant No. FA9550-20-1-0024. Virginia Selgas Visit to the University of Delaware funded by the program Movilidades de Excelencia of the University of Oviedo, and research partially supported by the project MTM2017-87162-P of MINECO.

\section{Author details}

'Department of Mathematical Sciences, University of Delaware, Newark 19716, USA, ²Departamento de Matemáticas, Universidad de Oviedo, EPIG, C/ Luis Ortiz Berrocal s/n, 33203 Gijón, Spain. 


\section{References}

1. Agranovich, M.S., Amosov, B.A., Levitin, M.:Spectral problems for the Lamé system with spectral parameter in boundary conditions on smooth or nonsmooth boundary. Russ. J. Math. Phys. 6, 247-281 (1999)

2. Audibert, L., Cakoni, F., Haddar, H.: New sets of eigenvalues in inverse scattering for inhomogeneous media and their determination from scattering data. Inverse Probl. 30, 125001 (2015)

3. Cakoni, F., Colton, D.: Qualitative Methods in Inverse Scattering Theory. Springer, New York (2006)

4. Cakoni, F., Colton, D., Meng, S., Monk, P.: Stekloff eigenvalues in inverse scattering. SIAM J. Appl. Math. 76(4), 1737-1763 (2016)

5. Camaño, J., Lackner, C., Monk, P.: Electromagnetic Stekloff eigenvalues in inverse Scattering. SIAM J. Math. Anal. 49 4376-4401 (2017)

6. Cogar, S.: A modified transmission eigenvalue problem in inverse scattering by a partially coated crack. Inverse Probl. 34, 115003 (2018)

7. Cogar, S., Colton, D., Leung, Y.-J.: The inverse spectral problem for transmission eigenvalues. Inverse Probl. $\mathbf{3 3}(5)$, 055015 (2017)

8. Cogar, S., Colton, D., Meng, S., Monk, P.: Modified transmission eigenvalues in inverse scattering theory. Inverse Probl. 33(12), $125002(2017)$

9. Cogar, S., Monk, P.: Modified electromagnetic transmission eigenvalues in inverse scattering theory. In preparation (2020)

10. Colton, D., Kress, R.: Inverse Acoustic and Electromagnetic Scattering Theory, 4th edn, Springer, New York (2019)

11. Colton, D., Monk, P.:A new method for solving the inverse scattering problem for acoustic waves in an inhomogeneous medium. Inverse Probl. 5, 1013-1026 (1989)

12. Colton, D., Monk, P.: A new method for solving the inverse scattering problem for acoustic waves in an inhomogeneous medium II. Inverse Probl. 6, 935-947 (1990)

13. Costabel, M., Stephan, E.: A direct boundary integral equation method for transmission problems. J. Math. Anal. Appl. 106, 367-413 (1985)

14. Elschner, J., Hsiao, G.C., Rathsfeld, A.: An inverse problem for fluid-solid interaction. Inverse Probl. Imag. 2, 83-119 (2008)

15. Friedlander, L.: Some inequalities between Dirichlet and Neumann eigenvalues. Arch. Rat. Mech. Anal. 116, 153-160 (1991)

16. Hsiao, G.C., Kleinman, R.E., Roach, G.F.: Weak solutions of fluid-solid interaction problems. Math. Nachr. 218, 139-163 (2000)

17. Kress, R.: Linear Integral Equations. Springer, Berlin (1989)

18. Kupradze, V.D., Gegelia, T.G., Basheleishvili, M.O., Burchuladze, T.V.: Three-Dimensional Problems of the Mathematical Theory of Elasticity and Thermoelasticity. Nauka, Moscow (1976); English transl., North-Holland, Amsterdam (1979)

19. Levitin, M., Marletta, M.: A simple method of calculating eigenvalues and resonances in domains with infinite regular ends. Proc. R. Soc. Edinb. Sect. A Math. 138A, 1043-1065 (2008)

20. Levitin, M., Monk, P., Selgas, V.: Impedance eigenvalues for inverse scattering in a fluid-solid interaction problem. In preparation (2021)

21. Luke, C.J., Martin, P.A.: Fluid-solid interaction: acoustic scattering by a smooth elastic obstacle. SIAM J. Appl. Math. 55 904-922 (1995)

22. Márquez, A., Meddahi, S., Selgas, V.: A new BEM-FEM coupling strategy for two-dimensional fluid-solid interaction problems. J. Comput. Phys. 199, 205-220 (2004)

23. Melrose, R.B.: Geometric Scattering Theory. Cambridge University Press, Cambridge (1995)

24. Monk, P., Selgas, V.: An inverse fluid-solid interaction problem. Inverse Probl. Imag. 3(2), 173-198 (2009)

25. Monk, P., Selgas, V.: Near field sampling type methods for the inverse fluid-solid interaction problem. Inverse Probl. Imag. 5, 465-483 (2011)

26. Morse, P.M., H. Feshbach, H.: Methods of Theoretical Physics, part II. McGraw-Hill, New York (1953)

27. Natroshvili, D., Kharibegashvili, S., Tediashvili, Z.: Direct and inverse fluid-structure interaction problems. Rend. Mat. Appl. (VII) 20, 57-92 (2000)

28. Schöberl, J.: Netgen/Ngsolv. https://ngsolve.org (2020)

29. Nguyen, H.-M.: Limiting absorption principle and well-posedness for the Helmholtz equation with sign changing coefficients. J. Math. Pures Appl. 106, 342-374 (2016)

\section{Publisher's Note}

Springer Nature remains neutral with regard to jurisdictional claims in published maps and institutional affiliations. 